\title{
Genetic Algorithms applied to Problems of Forbidden Configurations
}

\author{
R.P. Anstee* Miguel Raggi ${ }^{\dagger}$ \\ Department of Mathematics \\ University of British Columbia \\ Vancouver, B.C. Canada V6T 1Z2 \\ anstee@math.ubc.ca mraggi@gmail.com \\ Submitted: Jun 27, 2011; Accepted: Nov 15, 2011; Published: Dec 5, 2011 \\ Mathematics Subject Classification: 05C88, 05D05
}

\begin{abstract}
A simple matrix is a $(0,1)$-matrix with no repeated columns. For a $(0,1)$-matrix $F$, we say a $(0,1)$-matrix $A$ avoids $F$ (as a configuration) if there is no submatrix of $A$ which is a row and column permutation of $F$. Let $\|A\|$ denote the number of columns of $A$. We define $\operatorname{forb}(m, F)=\max \{\|A\|: A$ is an $m$-rowed simple matrix that avoids $F\}$. Define an extremal matrix as an $m$-rowed simple matrix $A$ with that avoids $F$ and $\|A\|=$ forb $(m, F)$. We describe the use of Local Search Algorithms (in particular a Genetic Algorithm) for finding extremal matrices. We apply this technique to two forbidden configurations in turn, obtaining a guess for the structure of an $m \times \operatorname{forb}(m, F)$ simple matrix avoiding $F$ and then proving the guess is indeed correct. The Genetic Algorithm was also helpful in finding the proof.
\end{abstract}

Keywords: trace, forbidden configurations, extremal set theory, (0,1)-matrices, Genetic Algorithms

\section{Introduction}

Define a matrix to be simple if it is a $(0,1)$-matrix with no repeated columns. Then an $m \times n$ simple matrix corresponds to a simple hypergraph or set system on $m$ vertices with $n$ edges. For a matrix $A$, let $\|A\|$ denote the number of columns in $A$. For a $(0,1)$-matrix $F$, we define that a $(0,1)$-matrix $A$ has $F$ as a configuration, which we write as $F \prec A$, if there is a submatrix of $A$ which is a row and column permutation of $F$. Let $\operatorname{Avoid}(m, F)$

\footnotetext{
* Research supported in part by NSERC

${ }^{\dagger}$ Research supported in part by NSERC of first author
} 
denote the set of all $m$-rowed simple matrices $A$ with $F \nprec A$. Our main extremal problem is to compute

$$
\operatorname{forb}(m, F)=\max _{A}\{\|A\|: A \in \operatorname{Avoid}(m, F)\} .
$$

We say a matrix $A \in \operatorname{Avoid}(m, F)$ is an extremal matrix if $\|A\|=\operatorname{forb}(m, F)$. We will also consider the natural generalizations where we forbid a family $\mathcal{F}$ of configurations.

In this paper we describe a new computational approach for finding extremal matrices using a Genetic Algorithm as described in Section 2. For a general introduction to Genetic Algorithms one could use [9], [10]. Genetic Algorithms have been applied in a number of Combinatorial problems such as Graph Colouring [6], Travelling Salesman Problem [8] and Steiner Tree Problem [7]. It is quite remarkable that this local search strategy has given such reliable results for us.

We study the following configurations $V$ and $W$ (where forb $(m, V)$ and $\operatorname{forb}(m, W)$ weren't previously known), and compare to the common submatrix $X$ (where forb $(m, X)$ was found in [2]).

$$
X=\left[\begin{array}{ll}
1 & 1 \\
1 & 1 \\
0 & 0
\end{array}\right], \quad V=\left[\begin{array}{llll}
1 & 1 & 0 & 0 \\
1 & 1 & 0 & 0 \\
0 & 0 & 1 & 1
\end{array}\right], \quad W=\left[\begin{array}{llll}
1 & 1 & 1 & 1 \\
1 & 1 & 0 & 0 \\
0 & 0 & 1 & 1
\end{array}\right]
$$

We use our Genetic Algorithm to seek extremal matrices $A \in \operatorname{Avoid}(m, V)$ and $A \in \operatorname{Avoid}(m, W)$ for small $m$. From these examples we guess the structure of extremal matrices in general and then we are subsequently able to prove these matrices are indeed extremal. Guessing such structures would have been challenging without the help of a computer. Matrix $X$ above is contained in both $V, W$ and we initially thought (before using the Genetic Algorithm) that forb $(m, V)=\operatorname{forb}(m, W)=\operatorname{forb}(m, X)$. Related results are in [2],[3]. In particular a list of $3 \times 4$ configurations for which the exact bounds are not known are listed in [3].

Theorem 1.1. [2] Let $m \geq 3$. Then forb $(m, X)=\left(\begin{array}{c}m \\ 2\end{array}\right)+\left(\begin{array}{c}m \\ 1\end{array}\right)+\left(\begin{array}{c}m \\ 0\end{array}\right)+1$.

Theorem 1.2. Let $m \geq 2$. Then forb $(m, W)=\left(\begin{array}{c}m \\ 2\end{array}\right)+\left(\begin{array}{c}m \\ 1\end{array}\right)+\left(\begin{array}{c}m \\ 0\end{array}\right)+m-2$.

Theorem 1.3. Let $m \geq 6$. Then forb $(m, V)=\left(\begin{array}{c}m \\ 2\end{array}\right)+\left(\begin{array}{c}m \\ 1\end{array}\right)+\left(\begin{array}{c}m \\ 0\end{array}\right)+3$.

The structure of the apparently extremal matrices $A$ generated by the Genetic Algorithm provided the strategy to tackle the latter two bounds. Interestingly, the Genetic Algorithm was used again in each example in the inductive proof to guess forb $(m-1,\{$ a collection of smaller matrices $\})$. We establish Theorem 1.2 in Section 4 and Theorem 1.3 in Section 5. Computer calculations were used during the course of the proof to compute 'what is missing?' on each four rows as described in Section 3. All code $(\mathrm{C}++)$ used in this paper can be freely downloaded from http://www.math.ubc.ca/ anstee/.

Some helpful notation is the following. Let $[m]=\{1,2, \ldots, m\}$. We say a column $\alpha$ has column sum $\ell$ if $\alpha$ has exactly $\ell 1$ 's. Let $K_{k}$ denote the $k \times 2^{k}$ simple matrix that consists of all $2^{k}$ possible columns in $k$ rows. Let $K_{k}^{\ell}$ denote a $k \times\left(\begin{array}{l}k \\ l\end{array}\right)$ simple matrix of all 
columns of sum $\ell$. Let $\mathbf{1}_{k}$ denote the $k \times 1$ column of 1 's and $\mathbf{0}_{\ell}$ denote the $\ell \times 1$ column of 0 's. For two given matrices $A, B$ which have the same number of rows, let $[A \mid B]$ denote the matrix of $A$ concatenated with $B$. Let $K_{k}^{\leq \ell}$ denote the matrix $\left[K_{k}^{\ell}\left|K_{k}^{\ell-1}\right| \cdots \mid K_{k}^{0}\right]$. For a set of rows $S$, let $\left.A\right|_{S}$ denote the submatrix of $A$ restricted to rows $S$.

We define $B \times C$ as the product of two matrices $B, C$ as follows: Assume $B, C$ have $b, c$ rows respectively. Then $B \times C$ has $b+c$ rows and $\|B\| \cdot\|C\|$ columns. The columns are formed from a column of $B$ on top of a column of $C$ in all possible ways. Column $\mathbf{1}_{2} \times \mathbf{0}_{2}$ would be $(1,1,0,0)^{T}$. Also [01] $\times K_{k-1}=K_{k}$. Product constructions were conjectured in [4] to be the asymptotically best constructions avoiding a configuration. A survey on Forbidden Configurations can be found in [1].

\section{Genetic Algorithm for Finding Extremal Matrices}

In this section, we consider $\mathcal{F}$ a family of (small) configurations and $m$ a (small) fixed integer. Suppose we wish to to find (or rather, guess) forb $(m, \mathcal{F})$ using the computer. The idea is to consider all the $2^{m}$ columns and add them one by one into a matrix $A \in \operatorname{Avoid}(m, \mathcal{F})$, making sure at each step we don't create any $F \in \mathcal{F}$ as a configuration. The order in which to add columns is what will determine the size of $\|A\|$ at the end of the process. To do this, enumerate all the columns and for each permutation of $\left[2^{m}\right]$ find the number of columns that would be added while avoiding all $F \in \mathcal{F}$ if we were to add them in that order. So the search space has size $\left(2^{m}\right)$ !, which is too big to search exhaustively, but local search can find the correct answer when $m$ isn't too large. We'll describe three methods.

For each permutation, we can separate the columns into good columns (which together form a matrix in $\operatorname{Avoid}(m, \mathcal{F})$ ) and bad columns. We wish then to find a permutation that maximizes the number of good columns. Perhaps this explanation could benefit by an example. Suppose we wanted to forbid

$$
F=\left[\begin{array}{ll}
1 & 0 \\
0 & 1
\end{array}\right]
$$

and suppose $m=3$. Consider all eight 3-rowed columns in the following order:

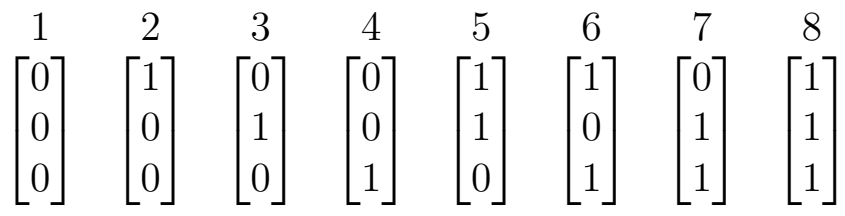

Then consider the permutation $(4,1,3,6,5,8,2,7)$. We start adding columns in the order given by this permutation. If adding a certain column would give rise to a copy of $F$, then don't add it and continue to the next one. Here is how it would work for this particular permutation: 
- Add column 4 .

$$
A=\left[\begin{array}{l}
0 \\
0 \\
1
\end{array}\right]
$$

- Add column 1:

$$
A=\left[\begin{array}{ll}
0 & 0 \\
0 & 0 \\
1 & 0
\end{array}\right]
$$

- Can't add column 3.

- Add column 6:

$$
A=\left[\begin{array}{lll}
0 & 0 & 1 \\
0 & 0 & 0 \\
1 & 0 & 1
\end{array}\right]
$$

- Can't add column 5.

- Add column 8:

$$
A=\left[\begin{array}{llll}
0 & 0 & 1 & 1 \\
0 & 0 & 0 & 1 \\
1 & 0 & 1 & 1
\end{array}\right]
$$

- Can't add column 2.

- Can't add column 7 .

We end up with four good columns, $\{4,1,6,8\}$ and four bad columns, $\{3,5,2,7\}$.

\subsection{Brute Force approach}

The brute force method does the following:

- Choose a permutation at random.

- Separate into good and bad columns.

- Count the number of good columns.

- Repeat, while keeping track of the "best" so far (i.e. the one with the most number of good columns)

The strength of this method is that we may do this thousands of times in a relatively short time. This method is good for small configurations in which there are many different ways to achieve the bound but, in our experience, there are many cases where this method fails to find an extremal matrix. 


\subsection{Hill Climbing}

This is an improvement over the above described method using the idea of Hill Climbing. Start with some permutation and separate into good and bad columns, and then for each column $b$ in $b a d$, form a new permutation by putting $b$ at the beginning, but leaving all others in place. This ensures that the chosen column $b$ is selected when performing the separation task again for the new permutation. From all possible choices of $b$, pick the "best one"; that is, the one which gives rise to the most columns in good. If there are ties, pick one at random. Repeat this process until there is no improvement for a number of steps. In the example described before, we would have to consider all the following permutations:

1. $(3,4,1,6,5,8,2,7)$

2. $(5,4,1,3,6,8,2,7)$

3. $(2,4,1,3,6,5,8,7)$

4. $(7,4,1,3,6,5,8,2)$

In this example all these permutations give four good columns, but in general some might be better than others.

Hill Climbing as described above gets stuck easily, but it does perform better than brute force.

\subsection{Genetic Algorithms}

An even better way (in practice, we've found) to search the space is using a Genetic Algorithm. The idea behind Genetic Algorithms is to mimic evolution. We maintain a population of 'individuals' and assign 'fitness' to them in some way (in our case, the number of 'good' columns). Then pick two at random, but giving higher probability to those with higher fitness. Call them father and mother. Then combine them in some way to produce offspring, hopefully with better results than either father or mother. Do this for many generations.

There are many ways to combine permutations. One that we found that works well for Forbidden Configurations is as follows:

1. For both father and mother, separate the numbers (of the columns) into good and bad.

2. Choose a random number from the good part of mother, and select all numbers (of columns) from the good part starting from the first good column and going up to the chosen number.

3. Permute the entries of father so that the numbers selected from the good part of mother are in the same order as those in mother. 
This is better shown with an example. Suppose we had a father and a mother like this:

$$
\text { father }=(2,3,6,1 \mid 7,4,8,5) \text { and mother }=(5,3,8,1,4 \mid 7,2,6)
$$

with the numbers shown before the vertical line being the good part and the numbers shown after the vertical line being the bad part. Pick a random entry in the good part of mother. For example, pick 1 and look at the numbers that appear to the left of the picked number. In this case, $(5,3,8,1)$. In father, select these numbers.

$$
\text { father }=(2, \mathbf{3}, 6, \mathbf{1} \mid 7,4, \mathbf{8}, \mathbf{5})
$$

Make child by shuffling the selected entries in father so that they match the order of mother.

$$
\text { child }=(2, \mathbf{5}, 6, \mathbf{3}, 7,4, \mathbf{8}, \mathbf{1}) .
$$

Finally, separate the columns of child into good and bad. This approach has given very good results in our experience. In particular for the two matrices $V$ and $W$ the Genetic Algorithm gave us the matrices which were later proved to be extremal. Then by having the conjectured extremal matrices it was relatively straightforward to construct the proofs. We discuss these proofs in the following sections.

\section{Two Proof techniques for Forbidden Configura- tions}

\subsection{Standard Induction}

The following induction idea has been very successful for studying Forbidden Configurations. Let $A \in \operatorname{Avoid}(m, F)$. Consider deleting a row $r$. Let $C_{r}(A)$ be the matrix that consists of the repeated columns of the matrix that is obtained when deleting row $r$ from $A$. For example, permute the rows of $A$ so that $r$ becomes the first row. Let $A^{\prime}$ be the matrix obtained from $A$ by deleting row $r$. Now $A^{\prime}$ need not be simple and so let $C_{r}(A)$ denote the largest simple $(m-1)$-rowed submatrix of $A^{\prime}$ so that $A^{\prime}$ contains $\left[C_{r}(A) C_{r}(A)\right]$. After column permutations, we may decompose $A$ as follows:

$$
A=\operatorname{row} r \rightarrow\left[\begin{array}{cccccc}
0 & \cdots & 0 & 1 & \cdots & 1 \\
B_{r}(A) & & C_{r}(A) & C_{r}(A) & & D_{r}(A)
\end{array}\right],
$$

where $B_{r}(A)$ are the columns that appear with a 0 on row $r$, but don't appear with a 1 , and $D_{r}(A)$ are the columns that appear with a 1 but not a 0 and $C_{r}(A)$ are the columns that appear with both with a 0 and with a 1 on row $r$. Thus $\left[B_{r}(A) C_{r}(A) D_{r}(A)\right]$ is a simple $(m-1)$-rowed matrix in $\operatorname{Avoid}(m-1, F)$. We deduce

$$
\|A\| \leq\left\|C_{r}(A)\right\|+\operatorname{forb}(m-1, F) .
$$

This means any upper bound on $\left\|C_{r}(A)\right\|$ (as a function of $m$ ), automatically yields an upper bound on $\|A\|$ by induction. 
Since $C_{r}(A)$ consists of the repeated columns, we can deduce extra conditions. In particular $A$ contains [ $\left[\begin{array}{ll}0 & 1\end{array}\right] \times C_{r}(A)$. Thus if for example $F^{\prime}$ is obtained from $F$ by deleting a row then $C_{r}(A) \in \operatorname{Avoid}\left(m-1, F^{\prime}\right)$. Also if $F \prec\left[F^{\prime \prime} \mid F^{\prime \prime}\right]$, then $C_{r} \in \operatorname{Avoid}\left(m-1, F^{\prime \prime}\right)$. We search for a row $r$ of $A$ such that $\left\|C_{r}(A)\right\|$ is as small as possible. If we could prove that there always exists a row $r$ such that $\left\|C_{r}(A)\right\|$ is small enough, we could proceed by induction.

\subsection{What is Missing?}

Suppose $F$, the forbidden configuration, has $s$ rows and suppose $A$ doesn't contain $F$. Consider all $s$-tuples of rows from $A$ and for each $s$-tuple of rows $S$, consider the submatrix $\left.A\right|_{S}$ of $A$ formed from rows $S$ of $A$. For example, if $s=2$ and $S=\{2,4\}$ and

$$
A=\left[\begin{array}{lllllll}
0 & 1 & 0 & 0 & 1 & 1 & 0 \\
0 & 1 & 1 & 0 & 1 & 0 & 0 \\
0 & 1 & 0 & 1 & 0 & 1 & 0 \\
0 & 0 & 0 & 1 & 0 & 1 & 1
\end{array}\right]
$$

then

$$
\left.A\right|_{S}=\left[\begin{array}{lllllll}
0 & 1 & 1 & 0 & 1 & 0 & 0 \\
0 & 0 & 0 & 1 & 0 & 1 & 1
\end{array}\right]
$$

Without any restriction, $A_{S}$ could have all $2^{|S|}$ possible columns, each appearing multiple times. But with the restriction that $F \nprec A$, in particular $\left.F \nprec A\right|_{S}$, so some columns have to be missing; we can't have all $2^{s}$ columns appear in $\left.A\right|_{S}$ as many times as they appear in $F$. Some may not appear at all. In our example, the column $\left[\begin{array}{l}0 \\ 0\end{array}\right]$ appears only once in $\left.A\right|_{S}$, while $\left[\begin{array}{l}1 \\ 0\end{array}\right]$ and $\left[\begin{array}{l}0 \\ 1\end{array}\right]$ both appear 3 times, but $\left[\begin{array}{l}1 \\ 1\end{array}\right]$ doesn't appear at all.

We wrote a program whose input is a configuration $F$ (or even a family of configurations $\mathcal{F}$ ), and its output is the list of possibilities for columns absent or appearing a restricted number of times. Studying this list often is often easier than studying $F$ for the purpose of analyzing what the structure of a matrix in $\operatorname{Avoid}(m, F)$ is. Unfortunately, the program performs doubly exponential $\left(O\left(2^{2^{s}}\right)\right)$ matrix comparison operations, where $s$ is the number of rows on which we wish to find what is missing. Furthermore, each matrix comparison takes $O(s !)$ time. In practice, this means checking $\leq 4$ rows is instantaneous, 5 rows takes, depending on the configuration, anywhere from a few minutes to a couple of hours, and with 6 rows we would have to make $\sim 2^{64}$ configuration comparison operations, which is many orders of magnitude more than any computer could handle.

\section{Exact bound for $W$}

We'll prove that forb $(m, W)=\left(\begin{array}{c}m \\ 2\end{array}\right)+\left(\begin{array}{c}m \\ 1\end{array}\right)+\left(\begin{array}{c}m \\ 0\end{array}\right)+m-2$. We can check that the first few cases (for $m \leq 5$ ) work using a computer. Then we proceed by the Standard Induction: Let $A \in \operatorname{Avoid}(m, F)$. If we could find some row $r$ for which $\left\|C_{r}(A)\right\| \leq m+1$, then we could conclude using 3.1 that 


$$
\begin{aligned}
\|A\| & \leq m+1+\left(\begin{array}{c}
m-1 \\
2
\end{array}\right)+\left(\begin{array}{c}
m-1 \\
1
\end{array}\right)+\left(\begin{array}{c}
m-1 \\
0
\end{array}\right)+(m-1)-2 \\
& =\left(\begin{array}{c}
m \\
2
\end{array}\right)+\left(\begin{array}{c}
m \\
1
\end{array}\right)+\left(\begin{array}{c}
m \\
0
\end{array}\right)+m-2 .
\end{aligned}
$$

So we may assume that $\left\|C_{r}(A)\right\| \geq m+2$ for every $r$.

Since $C_{r}(A)$ consists of repeated columns of $A$, note it cannot contain any of the following three configurations:

$$
G_{1}=\left[\begin{array}{ll}
1 & 1 \\
1 & 0 \\
0 & 1
\end{array}\right], \quad G_{2}=\left[\begin{array}{llll}
1 & 1 & 0 & 0 \\
0 & 0 & 1 & 1
\end{array}\right], \quad G_{3}=\left[\begin{array}{llll}
1 & 1 & 1 & 1 \\
1 & 1 & 0 & 0
\end{array}\right]
$$

Given that $C_{r}(A) \in \operatorname{Avoid}\left(m-1,\left\{G_{1}, G_{2}, G_{3}\right\}\right)$, the following Lemma establishes that $\left\|C_{r}(A)\right\| \leq(m-1)+3=m+2$.

Lemma 4.1. Let $m \geq 4$. Then forb $\left(m,\left\{G_{1}, G_{2}, G_{3}\right\}\right)=m+3$.

Proof: For the lower bound forb $\left(m,\left\{G_{1}, G_{2}, G_{3}\right\}\right) \geq m+3$ an example (which was found again using our Local Search strategies) suffices. Consider the matrix

$$
A=\left[\mathbf{0}_{m}\left|K_{m}^{1}\right| \mathbf{1}_{m} \mid \alpha\right],
$$

where $\alpha$ is any other column. Clearly $A \in \operatorname{Avoid}\left(m,\left\{G_{1}, G_{2}, G_{3}\right\}\right)$ and $\|A\|=m+3$. To prove the upper bound, we proceed by induction on $m$. Let $A \in \operatorname{Avoid}\left(m,\left\{G_{1}, G_{2}, G_{3}\right\}\right)$. Then, if we forbid $\left\{G_{1}, G_{2}, G_{3}\right\}$, below are the 16 possible cases of what is missing on each quadruple of rows.

$$
\begin{aligned}
& \text { no no no no no no no no no } \leq 1 \leq 1 \leq 1 \leq 1 \leq 1>1>1 \\
& {\left[\begin{array}{l}
1 \\
0 \\
1 \\
0
\end{array}\right]\left[\begin{array}{l}
0 \\
1 \\
1 \\
0
\end{array}\right]\left[\begin{array}{l}
1 \\
0 \\
0 \\
1
\end{array}\right]\left[\begin{array}{l}
0 \\
1 \\
0 \\
1
\end{array}\right]\left[\begin{array}{l}
1 \\
1 \\
0 \\
1
\end{array}\right]\left[\begin{array}{l}
0 \\
0 \\
1 \\
1
\end{array}\right]\left[\begin{array}{l}
1 \\
0 \\
1 \\
1
\end{array}\right]\left[\begin{array}{l}
0 \\
1 \\
1 \\
1
\end{array}\right]\left[\begin{array}{l}
1 \\
1 \\
1 \\
1
\end{array}\right]\left[\begin{array}{l}
1 \\
0 \\
0 \\
0
\end{array}\right]\left[\begin{array}{l}
0 \\
1 \\
0 \\
0
\end{array}\right]\left[\begin{array}{l}
0 \\
0 \\
1 \\
0
\end{array}\right]\left[\begin{array}{l}
1 \\
1 \\
1 \\
0
\end{array}\right]\left[\begin{array}{l}
0 \\
0 \\
0 \\
1
\end{array}\right]\left[\begin{array}{l}
0 \\
0 \\
0 \\
0
\end{array}\right]\left[\begin{array}{l}
1 \\
1 \\
0 \\
0
\end{array}\right]} \\
& \text { no no no no no no no no no } \leq 1 \leq 1 \leq 1 \leq 1 \leq 1>1>1 \\
& {\left[\begin{array}{l}
1 \\
0 \\
1 \\
0
\end{array}\right]\left[\begin{array}{l}
0 \\
1 \\
1 \\
0
\end{array}\right]\left[\begin{array}{l}
1 \\
1 \\
1 \\
0
\end{array}\right]\left[\begin{array}{l}
1 \\
0 \\
0 \\
1
\end{array}\right]\left[\begin{array}{l}
0 \\
1 \\
0 \\
1
\end{array}\right]\left[\begin{array}{l}
1 \\
1 \\
0 \\
1
\end{array}\right]\left[\begin{array}{l}
0 \\
0 \\
1 \\
1
\end{array}\right]\left[\begin{array}{l}
1 \\
0 \\
1 \\
1
\end{array}\right]\left[\begin{array}{l}
0 \\
1 \\
1 \\
1
\end{array}\right]\left[\begin{array}{l}
1 \\
0 \\
0 \\
0
\end{array}\right]\left[\begin{array}{l}
0 \\
1 \\
0 \\
0
\end{array}\right]\left[\begin{array}{l}
0 \\
0 \\
1 \\
0
\end{array}\right]\left[\begin{array}{l}
0 \\
0 \\
0 \\
1
\end{array}\right]\left[\begin{array}{l}
1 \\
1 \\
1 \\
1
\end{array}\right]\left[\begin{array}{l}
0 \\
0 \\
0 \\
0
\end{array}\right]\left[\begin{array}{l}
1 \\
1 \\
0 \\
0
\end{array}\right]} \\
& {\left[\begin{array}{l}
1 \\
1 \\
0 \\
0
\end{array}\right]\left[\begin{array}{l}
1 \\
0 \\
0 \\
1
\end{array}\right]\left[\begin{array}{l}
0 \\
1 \\
0 \\
1
\end{array}\right]\left[\begin{array}{l}
1 \\
0 \\
1 \\
0
\end{array}\right]\left[\begin{array}{l}
0 \\
1 \\
1 \\
0
\end{array}\right]\left[\begin{array}{l}
1 \\
1 \\
1 \\
0
\end{array}\right]\left[\begin{array}{l}
0 \\
0 \\
1 \\
1
\end{array}\right]\left[\begin{array}{l}
1 \\
0 \\
1 \\
1
\end{array}\right]\left[\begin{array}{l}
0 \\
1 \\
1 \\
1
\end{array}\right]\left[\begin{array}{l}
1 \\
0 \\
0 \\
0
\end{array}\right]\left[\begin{array}{l}
1 \\
1 \\
0 \\
0
\end{array}\right]\left[\begin{array}{l}
0 \\
0 \\
0 \\
1
\end{array}\right]\left[\begin{array}{l}
0 \\
0 \\
1 \\
0
\end{array}\right]\left[\begin{array}{l}
1 \\
1 \\
1 \\
1
\end{array}\right]\left[\begin{array}{l}
0 \\
0 \\
0 \\
0
\end{array}\right]\left[\begin{array}{l}
1 \\
1 \\
0 \\
1
\end{array}\right]}
\end{aligned}
$$


no no no no no no no no no no $\leq 1 \leq 1 \leq 1 \leq 1>1>1$

$[1][0][1][1][0][1][0][1][0][1][0][1][0][0][0][1]$

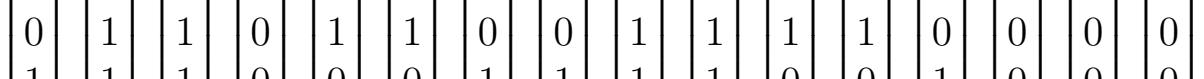

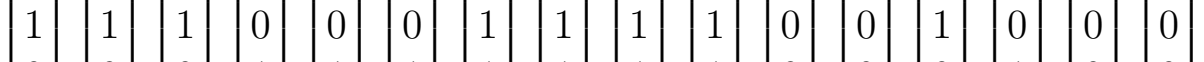

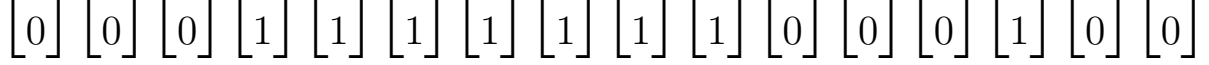

no no no no no no no no no no $\leq 1 \leq 1 \leq 1 \leq 1>1>1$ $[1][1][0][1][0][1][0][1][0][1]\left[\begin{array}{l}0 \\ {[0]}\end{array}\right][1][0][0][1]$ $\left.\left[\begin{array}{l}1 \\ 0 \\ 0\end{array}\right]\left[\begin{array}{l}0 \\ 1 \\ 0\end{array}\right]\left[\begin{array}{l}1 \\ 1 \\ 0\end{array}\right]\left[\begin{array}{l}0 \\ 0 \\ 1\end{array}\right]\left[\begin{array}{l}1 \\ 0 \\ 1\end{array}\right]\left[\begin{array}{l}1 \\ 0 \\ 1\end{array}\right]\left[\begin{array}{l}0 \\ 1 \\ 1\end{array}\right]\left[\begin{array}{l}0 \\ 1 \\ 1\end{array}\right]\left[\begin{array}{l}1 \\ 1 \\ 1\end{array}\right]\left[\begin{array}{l}1 \\ 1 \\ 1\end{array}\right]\left[\begin{array}{l}1 \\ 0 \\ 0\end{array}\right] \begin{array}{l}0 \\ 1 \\ 0\end{array}\right]\left[\begin{array}{l}1 \\ 1 \\ 0\end{array}\right]\left[\begin{array}{l}0 \\ 0 \\ 1\end{array}\right]\left[\begin{array}{l}0 \\ 0 \\ 0\end{array}\right]\left[\begin{array}{l}0 \\ 0 \\ 0\end{array}\right]$

no no no no no no no no no no $\leq 1 \leq 1 \leq 1 \leq 1>1>1$

$\left[\begin{array}{l}1 \\ 1\end{array}\right]\left[\begin{array}{l}1 \\ 0\end{array}\right]\left[\begin{array}{l}0 \\ 1\end{array}\right]\left[\begin{array}{l}1 \\ 1\end{array}\right]\left[\begin{array}{l}1 \\ 0\end{array}\right]\left[\begin{array}{l}0 \\ 1\end{array}\right]\left[\begin{array}{l}1 \\ 1\end{array}\right]\left[\begin{array}{l}0 \\ 0\end{array}\right]\left[\begin{array}{l}1 \\ 0\end{array}\right]\left[\begin{array}{l}0 \\ 1\end{array}\right]\left[\begin{array}{l}0 \\ 1\end{array}\right]\left[\begin{array}{l}0 \\ 0\end{array}\right]\left[\begin{array}{l}0 \\ 0\end{array}\right]\left[\begin{array}{l}1 \\ 1\end{array}\right]\left[\begin{array}{l}0 \\ 0\end{array}\right]\left[\begin{array}{l}1 \\ 0\end{array}\right]$ \begin{tabular}{llll|l|l|l|l|l|l|lll|lllllllll}
1 & 0 & 1 & 1 & 0 & 1 & 1 & 0 & 0 & 1 & 1 & 0 & 0 & 1 & 0 & 0
\end{tabular} $\left[\begin{array}{l}0 \\ 0\end{array}\right]\left[\begin{array}{l}1 \\ 0\end{array}\right]\left[\begin{array}{l}1 \\ 0\end{array}\right]\left[\begin{array}{l}1 \\ 0\end{array}\right]\left[\begin{array}{l}0 \\ 1\end{array}\right]\left[\begin{array}{l}0 \\ 1\end{array}\right]\left[\begin{array}{l}0 \\ 1\end{array}\right]\left[\begin{array}{l}1 \\ 1\end{array}\right]\left[\begin{array}{l}1 \\ 1\end{array}\right]\left[\begin{array}{l}1 \\ 1\end{array}\right]\left[\begin{array}{l}0 \\ 0\end{array}\right]\left[\begin{array}{l}1 \\ 0\end{array}\right]\left[\begin{array}{l}0 \\ 1\end{array}\right]\left[\begin{array}{l}1 \\ 1\end{array}\right]\left[\begin{array}{l}0 \\ 0\end{array}\right]\left[\begin{array}{l}0 \\ 0\end{array}\right]$ no no no no no no no no no no $\leq 1 \leq 1 \leq 1 \leq 1>1>1$ $[1][1][0][1][1][0][1][0][1][0][1][0][0][0][0][1]$ $\begin{array}{llllllllllllllllllllllllllll}1 & 0 & 1 & 1 & 0 & 1 & 1 & 0 & 0 & 1 & 0 & 1 & 0 & 0 & 0 & 1\end{array}$

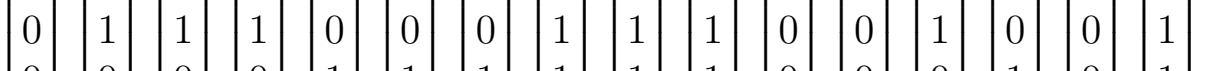

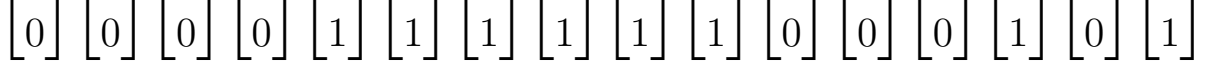

no no no no no no no no no no $\leq 1 \leq 1 \leq 1 \leq 1>1>1$ $[0][1][0][1][0][1][0][1][1][0]\left[\begin{array}{l}1 \\ {[0}\end{array}\right]\left[\begin{array}{l}0 \\ 0\end{array}\right]\left[\begin{array}{l}1 \\ 1\end{array}\right][0][1]$

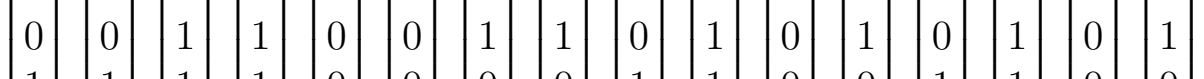

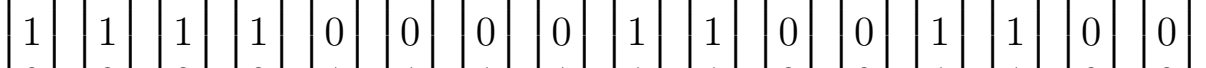

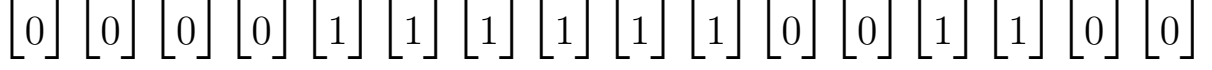

no no no no no no no no no no $\leq 1 \leq 1 \leq 1 \leq 1>1>1$ $[1][0][1][0][1][0][1][0][1][0][1][0][0][1][0][1]$ $\left[\begin{array}{l}1 \\ 0 \\ 0\end{array}\right]\left[\begin{array}{l}0 \\ 1 \\ 0\end{array}\right]\left[\begin{array}{l}0 \\ 1 \\ 0\end{array}\right]\left[\begin{array}{l}1 \\ 1 \\ 0\end{array}\right]\left[\begin{array}{l}1 \\ 1 \\ 0\end{array}\right]\left[\begin{array}{l}0 \\ 0 \\ 1\end{array}\right]\left[\begin{array}{l}0 \\ 0 \\ 1\end{array}\right]\left[\begin{array}{l}1 \\ 0 \\ 1\end{array}\right]\left[\begin{array}{l}1 \\ 0 \\ 1\end{array}\right]\left[\begin{array}{l}1 \\ 1 \\ 1\end{array}\right]\left[\begin{array}{l}0 \\ 0 \\ 0\end{array}\right]\left[\begin{array}{l}1 \\ 0 \\ 0\end{array}\right]\left[\begin{array}{l}0 \\ 1 \\ 1\end{array}\right]\left[\begin{array}{l}1 \\ 1 \\ 1\end{array}\right]\left[\begin{array}{l}0 \\ 0 \\ 0\end{array}\right]\left[\begin{array}{l}0 \\ 1 \\ 1\end{array}\right]$

no no no no no no no no no no no $\leq 1 \leq 1 \leq 1>1>1$

$[0][1][0][1][0][1][0][1][1][0][1][0][1][0][0][1]$

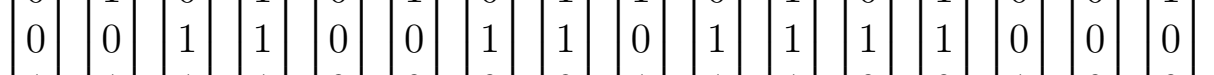
$\left[\begin{array}{l}1 \\ 0\end{array}\right]\left[\begin{array}{l}1 \\ 0\end{array}\right]\left[\begin{array}{l}1 \\ 0\end{array}\right]\left[\begin{array}{l}1 \\ 0\end{array}\right]\left[\begin{array}{l}0 \\ 1\end{array}\right]\left[\begin{array}{l}0 \\ 1\end{array}\right]\left[\begin{array}{l}0 \\ 1\end{array}\right]\left[\begin{array}{l}0 \\ 1\end{array}\right]\left[\begin{array}{l}1 \\ 1\end{array}\right]\left[\begin{array}{l}1 \\ 1\end{array}\right]\left[\begin{array}{l}1 \\ 1\end{array}\right]\left[\begin{array}{l}0 \\ 0\end{array}\right]\left[\begin{array}{l}0 \\ 0\end{array}\right]\left[\begin{array}{l}1 \\ 1\end{array}\right]\left[\begin{array}{l}0 \\ 0\end{array}\right]\left[\begin{array}{l}0 \\ 0\end{array}\right]$ no no no no no no no no no no no $\leq 1 \leq 1 \leq 1>1>1$ $[1][0][1][0][1][0][1][0][1][0][1][0][0][1][0][1]$

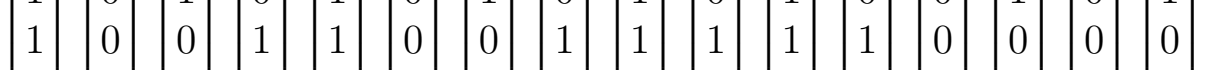

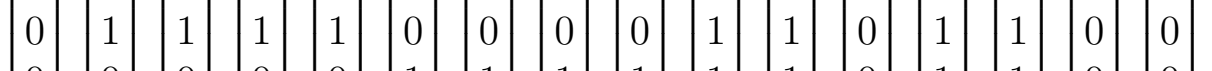

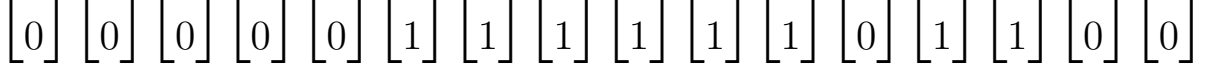




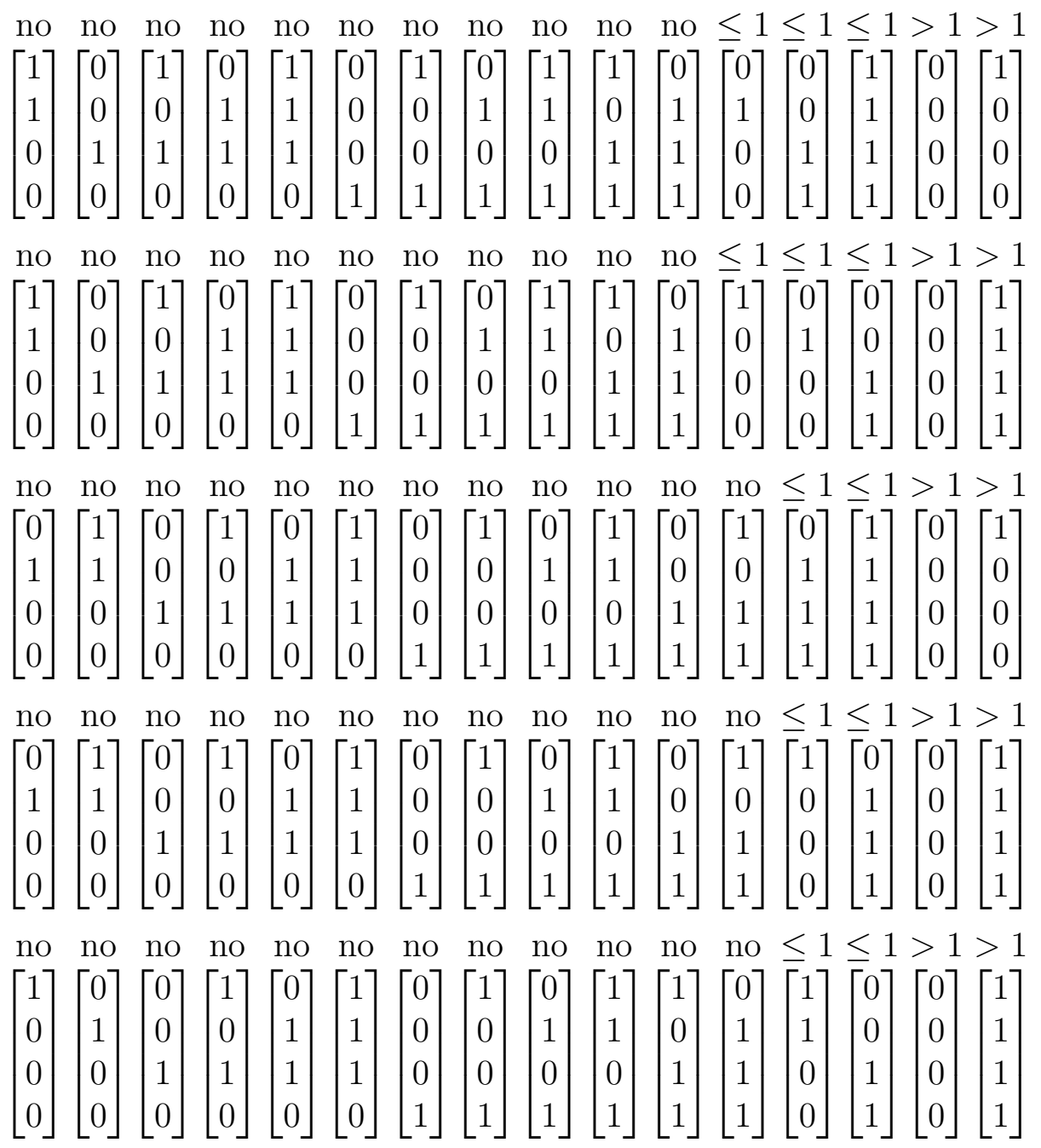

We easily check this list to see that there are at most seven columns present on four rows (at least 9 are absent) and so forb $\left(4,\left\{G_{1}, G_{2}, G_{3}\right\}\right)=7$. Now consider $m \geq 5$. It's easy to check that in every quadruple there is a row (the final row will always work) and a column we can delete from $A$ and keep the remaining $(m-1)$-rowed matrix $A^{\prime}$ simple. Then by induction, $\left\|A^{\prime}\right\| \leq(m-1)+3=m+2$ and then $\|A\| \leq\left\|A^{\prime}\right\|+1 \leq m+3$. To find such a row and column, look at the columns marked $\leq 1$ and $>1$, and see that there is a row we can delete such that the only repeat (if there is one) has one of the columns marked $\leq 1$. We used the help of the free software sage (http://www.sagemath.org/), but it could also be done by hand. For example, for the first possibility,

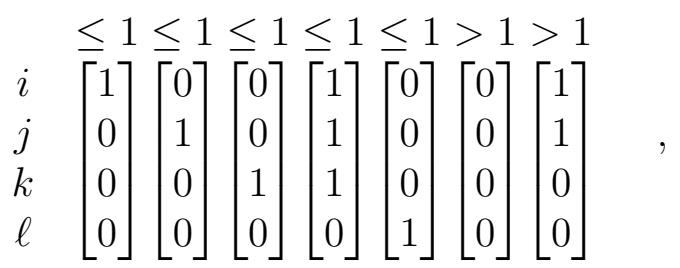

deleting from $A$ the row $\ell$ and the column of $A$ corresponding to the fifth column above is enough to keep the resulting matrix $A^{\prime}$ simple. Thus $\|A\| \leq m+3$. 
We need a more detailed lemma about the structure of any $m \times(m+3)$ matrix in $\operatorname{Avoid}\left(m,\left\{G_{1}, G_{2}, G_{3}\right\}\right)$. This was predicted using our Genetic Algorithm too.

Lemma 4.2. Let $A \in \operatorname{Avoid}\left(m,\left\{G_{1}, G_{2}, G_{3}\right\}\right)$ with $m \geq 5$ and $\|A\|=m+3$. Then $K_{m}^{1} \prec A$. Moreover, the remaining three columns are $\mathbf{0}_{m}$ and two additional columns $\alpha, \beta$ with $\alpha<\beta$ (meaning that on each row for which $\alpha$ has a 1, $\beta$ also has a 1 ).

Proof: We proceed by induction on $m$. We checked all cases with $m \leq 5$ using a computer. Assume $A \in \operatorname{Avoid}\left(m,\left\{G_{1}, G_{2}, G_{3}\right\}\right)$ with $\|A\|=m+3$ and $m \geq 6$. The fact that $A$ has no $G_{1}$ means the columns, when considered as sets, form a laminar family: Namely for any two columns $\alpha, \beta$, either $\alpha<\beta$ or $\beta<\alpha$ or $\alpha, \beta$ have no row in which both have 1's. Sometimes the term noncrossing is used. Viewing the columns as sets, we are asking that for any two sets $A, B$, either $A \subset B, B \subset A$ or $A \cap B=\emptyset$.

From our proof of Lemma 4.1 , there is a row and a column from $A$ we can delete to obtain a $(m-1) \times(m+2)$ simple matrix $A^{\prime}$. We may assume $K_{m-1}^{1} \prec A^{\prime}$ by induction. Assume we deleted the last row from $A$ to obtain $A^{\prime}$ and that the deleted column was the last column of $A$. If we restrict ourselves to the first $m-1$ rows, the deleted column has to be a repeat of one of the columns of $A^{\prime}$, else we would have an $(m-1) \times(m+3)$ simple matrix contradicting Lemma 4.1.

At this point the proof is finished save for a case analysis on each of the possible columns to repeat. In each case we repeat a column and attempt to find either $G_{1}, G_{2}$ or $G_{3}$. This case analysis is probably much easier as an exercise for the reader than it is to either write or read about. Given that no interesting ideas appear in this admittedly tedious but very straightforward case analysis, we have moved it to Appendix A for the interested reader.

We are now ready to prove Theorem 1.2.

Proof of Theorem 1.2: We use induction on $m$. The result is true for $m=3$ so we may assume $m \geq 4$. Let $A \in \operatorname{Avoid}(m, W)$. Apply the decomposition (3.1). If $\left\|C_{r}(A)\right\| \leq$ $m+1$, then we apply (3.1) to establish the bound for $\|A\|$ by induction. So assume $\left\|C_{r}(A)\right\| \geq m+2$ for all choices of $r$. By Lemma 4.1 , we deduce that $\left\|C_{r}(A)\right\|=m+2$ for every row $r$, and by Lemma 4.2, we have that $K_{m-1}^{1} \prec C_{r}(A)$ also for every row $r$. Thus $K_{m}^{2} \prec A$, since all columns of column sum 1 in $C_{r}(A)$ appear with a 1 in row $r$ (and this happens for every row $r$ ). We also have $\left[K_{m}^{1} \mid K_{m}^{0}\right] \prec A$. Now in every triple of rows of $K_{m}^{2}$ we have the matrix $G_{1}$ once in every ordering of the triple. Given that $W=\left[G_{1} \mid G_{1}\right]$, the columns of $A$ of column sum at least 3 have no configuration $G_{1}$. We appeal to Lemma 4.3 below to deduce that the number of columns in $A$ of column sum at least 3 is at most $m-2$. Then $\|A\| \leq\left(\begin{array}{c}m \\ 2\end{array}\right)+\left(\begin{array}{c}m \\ 1\end{array}\right)+\left(\begin{array}{c}m \\ 0\end{array}\right)+m-2$ which yields the desired bound.

A quick counting argument reveals the following general result about laminar families which we use in the proof of Theorem 1.2.

Lemma 4.3. Let $m \geq 3$ and let $\mathcal{Z}$ be a laminar family of subsets of $[m]=\{1,2, \ldots, m\}$ with the property that for all $Z \in \mathcal{Z}$ we have $|Z| \geq 3$. Then $|\mathcal{Z}| \leq m-2$ and furthermore, 
this bound is tight (i.e. there exist a family $\mathcal{Z}$ for which $|\mathcal{Z}|=m-2$ ). Thus, if $A \in$ $\operatorname{Avoid}(m, F)$ satisfies that all column sums are at least 3, then $\|A\| \leq m-2$.

Proof: Let $f(x)$ denote the size of the largest laminar family of $[x]$ with no sets of size less than or equal to 2. Assume $A \in \operatorname{Avoid}(m, F)$ with the property that all column sums are at least 3 . We wish to show that $f(m)=m-2$.

The family $\{[3],[4], \ldots,[m]\}$ has size $m-2$, which proves $f(m) \geq m-2$. We wish to prove $f(m) \leq m-2$.

Let $\mathcal{Z}$ be such that $|\mathcal{Z}|=f(m)$. We may assume $[m] \in \mathcal{Z}$ : if $[m] \notin \mathcal{Z}$, observe that $\mathcal{Z} \cup\{[m]\}$ is also a laminar family of bigger size. Suppose then that the next biggest set $Z$ in $\mathcal{Z}$ has size $k$. We partition $[m]$ into two disjoint sets: $Z$ and $[m] \backslash Z$. Every set $Y \in \mathcal{Z}$ satisfies either $Y \subseteq Z$ or $Y \subseteq[m] \backslash Z$ or $Y=[m]$. This gives the recurrence

$$
f(m) \leq 1+f(k)+f(m-k) .
$$

If $k \neq m-1$, then by induction $f(k)=k-2$ and $f(m-k)=m-k-2$, and so $f(m) \leq 1+k-2+m-k-2=m-3$, a contradiction. When $k=m-1$ we have $f(m) \leq m-2$. Moreover if $f(m)=m-2$ we observe that $\mathcal{Z}$ is "equivalent" to $\{[3],[4], \ldots,[m]\}$.

\section{Exact bound for $V$}

Using the computer, we can prove by exhaustively looking at all the possibilities that forb $(3, V)=8$, forb $(4, V)=13$, forb $(5, V)=18$. Using the Genetic Algorithm of Section 2, we obtained large matrices with no subconfiguration $V$ which suggested to us that forb $(6, V)=25$, forb $(7, V)=32$, forb $(8, V)=40$. For $m \geq 6$, this suggests forb $(m, V)=$ $\left(\begin{array}{c}m \\ 2\end{array}\right)+\left(\begin{array}{c}m \\ 1\end{array}\right)+\left(\begin{array}{c}m \\ 0\end{array}\right)+3$, two more than our first guess that forb $(m, V)=\operatorname{forb}(m, X)$.

The Genetic Algorithm also predicted the structure of matrices in $\operatorname{ext}(m, V)$. Following from the matrices discovered by the Genetic Algorithm, we will consider the following matrices in $\operatorname{Avoid}(m, V)$ : Consider a partition of the $m$ rows into two disjoint sets, say $U=\{1,2, \ldots, u\}$ and $L=\{u+1, u+2, \ldots, m\}$. Suppose $|U|=u$ and $|L|=\ell$ with $u+\ell=m$. Let $A^{\prime}$ have the following structure:

$$
A^{\prime}=\begin{gathered}
U \\
L
\end{gathered}\left[\begin{array}{c|c|c|c|c|c|c|c|c}
\mathbf{0}_{u} & \mathbf{0}_{u} & \mathbf{0}_{u} & \mathbf{0}_{u} & K_{u}^{1} & \mathbf{1}_{u} & K_{u}^{1} & K_{u}^{2} & \mathbf{1}_{u} \\
\times & \times & \times & \times & \times & \times & \times & \times & \times \\
\mathbf{0}_{\ell} & \mathbf{1}_{\ell} & K_{\ell}^{\ell-1} & K_{\ell}^{\ell-2} & K_{\ell}^{\ell-1} & \mathbf{0}_{\ell} & \mathbf{1}_{\ell} & \mathbf{1}_{\ell} & \mathbf{1}_{\ell}
\end{array}\right] .
$$

We easily check that $A^{\prime} \in \operatorname{Avoid}(m, V)$ and for $3 \leq u, \ell \leq m-3$ we have $\|A\|=$ $\left(\begin{array}{c}m \\ 2\end{array}\right)+\left(\begin{array}{c}m \\ 1\end{array}\right)+\left(\begin{array}{c}m \\ 0\end{array}\right)+3$. We will prove that $A^{\prime} \in \operatorname{ext}(m, V)$ and hence establish Theorem 1.3. To prove this, consider some $A \in \operatorname{Avoid}(m, V)$ and apply the standard decomposition 3.1. We deduce that $C_{r}(A)$ can't have any of the three following configurations:

$$
H_{1}=\left[\begin{array}{ll}
1 & 0 \\
1 & 0 \\
0 & 1
\end{array}\right], \quad H_{2}=\left[\begin{array}{llll}
1 & 1 & 0 & 0 \\
0 & 0 & 1 & 1
\end{array}\right], \quad H_{3}=\left[\begin{array}{cccc}
1 & 1 & 0 & 0 \\
1 & 1 & 0 & 0
\end{array}\right]
$$


We use the computer again to conjecture a structure on the $(m-1)$-rowed $C_{r}(A)$ when $\left\|C_{r}(A)\right\|=m+1$ and $C_{r}(A) \in \operatorname{Avoid}\left(m-1,\left\{H_{1}, H_{2}, H_{3}\right\}\right)$.

Lemma 5.1. Let $m \geq 4$ and $A \in \operatorname{Avoid}\left(m,\left\{H_{1}, H_{2}, H_{3}\right\}\right)$. We have $\|A\| \leq m+2$.

Proof: Using the computer, we find that one of the following must hold for each quadruple of rows of $A$.

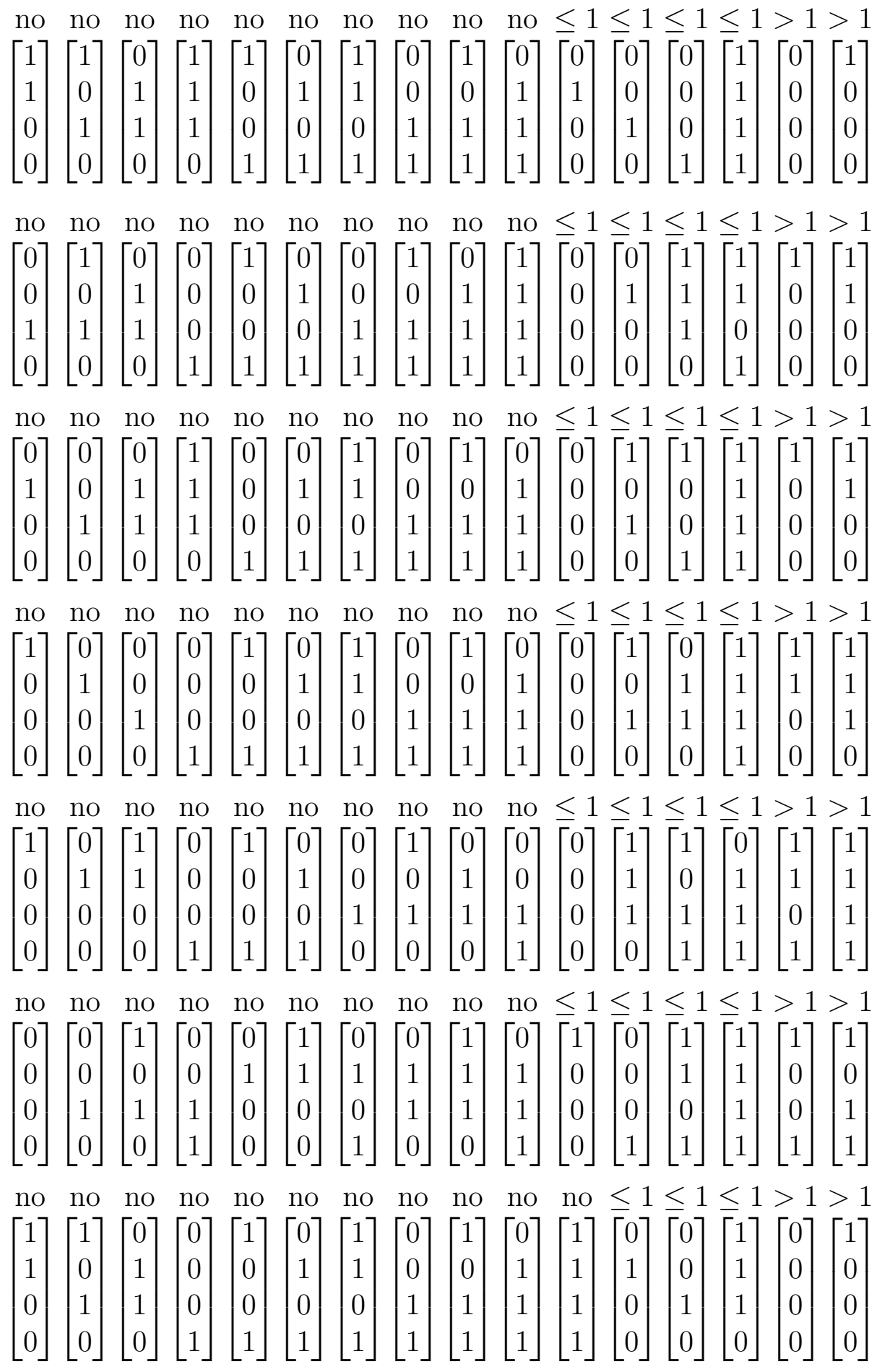


no no no no no no no no no no no $\leq 1 \leq 1 \leq 1>1>1$

$\left[\begin{array}{l}0 \\ 1 \\ 0 \\ 0\end{array}\right]\left[\begin{array}{l}0 \\ 0 \\ 1 \\ 0\end{array}\right]\left[\begin{array}{l}0 \\ 1 \\ 1 \\ 0\end{array}\right]\left[\begin{array}{l}1 \\ 1 \\ 1 \\ 0\end{array}\right]\left[\begin{array}{l}0 \\ 0 \\ 0 \\ 1\end{array}\right]\left[\begin{array}{l}0 \\ 1 \\ 0 \\ 1\end{array}\right]\left[\begin{array}{l}1 \\ 1 \\ 0 \\ 1\end{array}\right]\left[\begin{array}{l}0 \\ 0 \\ 1 \\ 1\end{array}\right]\left[\begin{array}{l}1 \\ 0 \\ 1 \\ 1\end{array}\right]\left[\begin{array}{l}0 \\ 1 \\ 1 \\ 1\end{array}\right]\left[\begin{array}{l}1 \\ 1 \\ 1 \\ 1\end{array}\right]\left[\begin{array}{l}1 \\ 1 \\ 0 \\ 0\end{array}\right]\left[\begin{array}{l}1 \\ 0 \\ 1 \\ 0\end{array}\right]\left[\begin{array}{l}1 \\ 0 \\ 0 \\ 1\end{array}\right]\left[\begin{array}{l}0 \\ 0 \\ 0 \\ 0\end{array}\right]\left[\begin{array}{l}1 \\ 0 \\ 0 \\ 0\end{array}\right]$

no no no no no no no no no no no $\leq 1 \leq 1 \leq 1>1>1$
$\left[\begin{array}{l}0 \\ 0 \\ 1 \\ 0\end{array}\right]\left[\begin{array}{l}1 \\ 0 \\ 1 \\ 0\end{array}\right]\left[\begin{array}{l}0 \\ 1 \\ 1 \\ 0\end{array}\right]\left[\begin{array}{l}1 \\ 1 \\ 1 \\ 0\end{array}\right]\left[\begin{array}{l}0 \\ 0 \\ 0 \\ 1\end{array}\right]\left[\begin{array}{l}1 \\ 0 \\ 0 \\ 1\end{array}\right]\left[\begin{array}{l}0 \\ 1 \\ 0 \\ 1\end{array}\right]\left[\begin{array}{l}1 \\ 1 \\ 0 \\ 1\end{array}\right]\left[\begin{array}{l}0 \\ 0 \\ 1 \\ 1\end{array}\right]\left[\begin{array}{l}1 \\ 0 \\ 1 \\ 1\end{array}\right]\left[\begin{array}{l}0 \\ 1 \\ 1 \\ 1\end{array}\right]\left[\begin{array}{l}0 \\ 0 \\ 0 \\ 0\end{array}\right]\left[\begin{array}{l}0 \\ 1 \\ 0 \\ 0\end{array}\right]\left[\begin{array}{l}1 \\ 1 \\ 1 \\ 1\end{array}\right]\left[\begin{array}{l}1 \\ 0 \\ 0 \\ 0\end{array}\right]\left[\begin{array}{l}1 \\ 1 \\ 0 \\ 0\end{array}\right]$

no no no no no no no no no no no $<1<1<1>1>1$

$[0][0][0][0][1][0][1][0][1][0][1][0][1][1][1][1]$

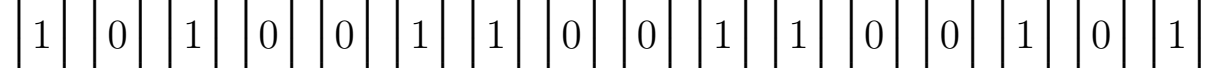
$\left[\begin{array}{l}0 \\ 0\end{array}\right]\left[\begin{array}{l}1 \\ 0\end{array}\right]\left[\begin{array}{l}1 \\ 0\end{array}\right]\left[\begin{array}{l}0 \\ 1\end{array}\right]\left[\begin{array}{l}0 \\ 1\end{array}\right]\left[\begin{array}{l}0 \\ 1\end{array}\right]\left[\begin{array}{l}0 \\ 1\end{array}\right]\left[\begin{array}{l}1 \\ 1\end{array}\right]\left[\begin{array}{l}1 \\ 1\end{array}\right]\left[\begin{array}{l}1 \\ 1\end{array}\right]\left[\begin{array}{l}1 \\ 1\end{array}\right]\left[\begin{array}{l}0 \\ 0\end{array}\right]\left[\begin{array}{l}1 \\ 0\end{array}\right]\left[\begin{array}{l}1 \\ 0\end{array}\right]\left[\begin{array}{l}0 \\ 0\end{array}\right]\left[\begin{array}{l}0 \\ 0\end{array}\right]$ no no no no no no no no no no no $\leq 1 \leq 1 \leq 1>1>1$ $[1][0][0][1][0][0][1][0][0][1][0][0][1][1][1][1]$

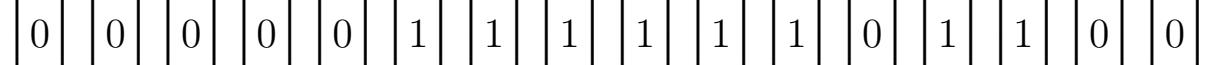

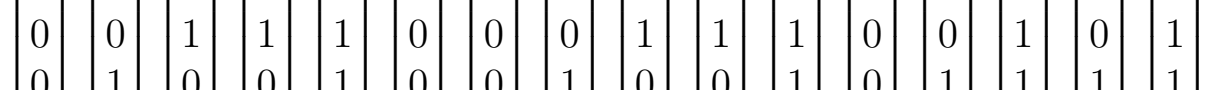

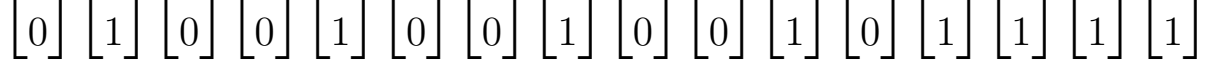

no no no no no no no no no no no $\leq 1 \leq 1 \leq 1>1>1$ $\left[\begin{array}{l}0 \\ 0 \\ 0 \\ 0\end{array}\right]\left[\begin{array}{l}0 \\ 1 \\ 0 \\ 0\end{array}\right]\left[\begin{array}{l}0 \\ 0 \\ 1 \\ 0\end{array}\right]\left[\begin{array}{l}0 \\ 1 \\ 1 \\ 0\end{array}\right]\left[\begin{array}{l}0 \\ 0 \\ 0 \\ 1\end{array}\right]\left[\begin{array}{l}1 \\ 0 \\ 0 \\ 1\end{array}\right]\left[\begin{array}{l}0 \\ 1 \\ 0 \\ 1\end{array}\right]\left[\begin{array}{l}1 \\ 1 \\ 0 \\ 1\end{array}\right]\left[\begin{array}{l}0 \\ 0 \\ 1 \\ 1\end{array}\right]\left[\begin{array}{l}1 \\ 0 \\ 1 \\ 1\end{array}\right]\left[\begin{array}{l}0 \\ 1 \\ 1 \\ 1\end{array}\right]\left[\begin{array}{l}1 \\ 0 \\ 0 \\ 0\end{array}\right]\left[\begin{array}{l}1 \\ 0 \\ 1 \\ 0\end{array}\right]\left[\begin{array}{l}1 \\ 1 \\ 1 \\ 1\end{array}\right]\left[\begin{array}{l}1 \\ 1 \\ 0 \\ 0\end{array}\right]\left[\begin{array}{l}1 \\ 1 \\ 1 \\ 0\end{array}\right]$

no no no no no no no no no no no $\leq 1 \leq 1 \leq 1>1>1$ $[0][0][1][0][1][0][0][1][0][0][0][1][1][1][1][1]$ $\left[\begin{array}{l}0 \\ 0 \\ 0\end{array}\right]\left[\begin{array}{l}1 \\ 0 \\ 0\end{array}\right]\left[\begin{array}{l}1 \\ 0 \\ 0\end{array}\right]\left[\begin{array}{l}0 \\ 0 \\ 1\end{array}\right]\left[\begin{array}{l}0 \\ 0 \\ 1\end{array}\right]\left[\begin{array}{l}1 \\ 0 \\ 1\end{array}\right]\left[\begin{array}{l}0 \\ 1 \\ 0\end{array}\right]\left[\begin{array}{l}0 \\ 1 \\ 0\end{array}\right]\left[\begin{array}{l}1 \\ 1 \\ 0\end{array}\right]\left[\begin{array}{l}0 \\ 1 \\ 1\end{array}\right]\left[\begin{array}{l}1 \\ 1 \\ 1\end{array}\right]\left[\begin{array}{l}0 \\ 0 \\ 0\end{array}\right]\left[\begin{array}{l}1 \\ 1 \\ 0\end{array}\right]\left[\begin{array}{l}0 \\ 1 \\ 1\end{array}\right]\left[\begin{array}{l}1 \\ 0 \\ 1\end{array}\right]\left[\begin{array}{l}1 \\ 1 \\ 1\end{array}\right]$ no no no no no no no no no no no $\leq 1 \leq 1 \leq 1>1>1$
$\left[\begin{array}{l}0 \\ 0 \\ 0 \\ 0\end{array}\right]\left[\begin{array}{l}1 \\ 0 \\ 0 \\ 0\end{array}\right]\left[\begin{array}{l}0 \\ 1 \\ 0 \\ 0\end{array}\right]\left[\begin{array}{l}0 \\ 0 \\ 0 \\ 1\end{array}\right]\left[\begin{array}{l}0 \\ 0 \\ 1 \\ 0\end{array}\right]\left[\begin{array}{l}1 \\ 0 \\ 1 \\ 0\end{array}\right]\left[\begin{array}{l}0 \\ 1 \\ 1 \\ 0\end{array}\right]\left[\begin{array}{l}1 \\ 1 \\ 1 \\ 0\end{array}\right]\left[\begin{array}{l}0 \\ 0 \\ 1 \\ 1\end{array}\right]\left[\begin{array}{l}1 \\ 0 \\ 1 \\ 1\end{array}\right]\left[\begin{array}{l}0 \\ 1 \\ 1 \\ 1\end{array}\right]\left[\begin{array}{l}1 \\ 1 \\ 0 \\ 0\end{array}\right]\left[\begin{array}{l}1 \\ 0 \\ 0 \\ 1\end{array}\right]\left[\begin{array}{l}0 \\ 1 \\ 0 \\ 1\end{array}\right]\left[\begin{array}{l}1 \\ 1 \\ 0 \\ 1\end{array}\right]\left[\begin{array}{l}1 \\ 1 \\ 1 \\ 1\end{array}\right]$ no no no no no no no no no no no no $\leq 1 \leq 1>1>1$ $\left[\begin{array}{l}0 \\ 0 \\ 1 \\ 0\end{array}\right]\left[\begin{array}{l}1 \\ 0 \\ 1 \\ 0\end{array}\right]\left[\begin{array}{l}0 \\ 1 \\ 1 \\ 0\end{array}\right]\left[\begin{array}{l}1 \\ 1 \\ 1 \\ 0\end{array}\right]\left[\begin{array}{l}0 \\ 0 \\ 0 \\ 1\end{array}\right]\left[\begin{array}{l}1 \\ 0 \\ 0 \\ 1\end{array}\right]\left[\begin{array}{l}0 \\ 1 \\ 0 \\ 1\end{array}\right]\left[\begin{array}{l}1 \\ 1 \\ 0 \\ 1\end{array}\right]\left[\begin{array}{l}0 \\ 0 \\ 1 \\ 1\end{array}\right]\left[\begin{array}{l}1 \\ 0 \\ 1 \\ 1\end{array}\right]\left[\begin{array}{l}0 \\ 1 \\ 1 \\ 1\end{array}\right]\left[\begin{array}{l}1 \\ 1 \\ 1 \\ 1\end{array}\right]\left[\begin{array}{l}0 \\ 1 \\ 0 \\ 0\end{array}\right]\left[\begin{array}{l}1 \\ 1 \\ 0 \\ 0\end{array}\right]\left[\begin{array}{l}0 \\ 0 \\ 0 \\ 0\end{array}\right]\left[\begin{array}{l}1 \\ 0 \\ 0 \\ 0\end{array}\right]$ 


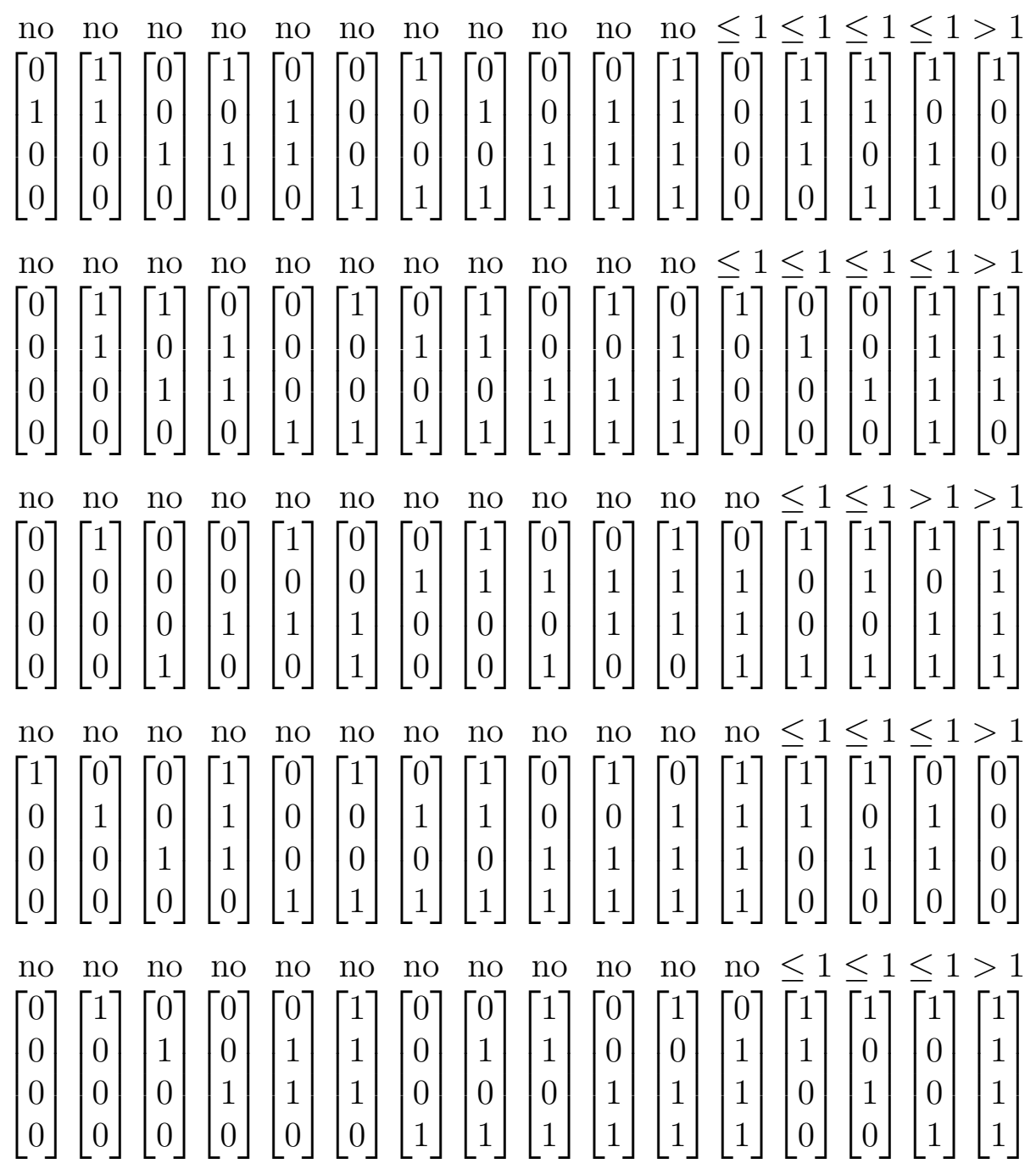

This verifies in particular that forb $\left(4,\left\{H_{1}, H_{2}, H_{3}\right\}\right)=6$ (at least 10 columns out of 16 are absent in each of the twenty cases). We proceed as we did in Lemma 4.1 and verify that each of these possibilities yields one row and at most one column that we can delete from $A$, maintaining simplicity. This means that $\|A\| \leq m+2$ by induction.

Lemma 5.2. Assume $m \geq 4$. Let $A \in \operatorname{Avoid}\left(m,\left\{H_{1}, H_{2}, H_{3}\right\}\right)$ with $\|A\|=m+2$. Then there exists a partition $U \subseteq[m]$ and $L=[m] \backslash U$ with $|U|=u \geq 1$ and $|L|=\ell=m-u \geq 1$ ( $U$ for Upper and $L$ for Lower) so that if we permute rows,

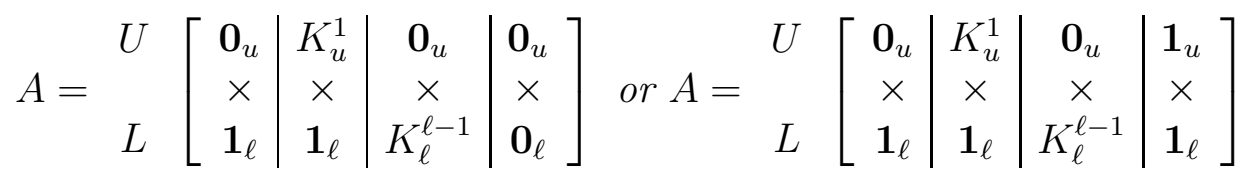

Note that in the former case we must have $\ell \geq 2$ and in the latter case $u \geq 2$.

Proof: We use induction on $m$. The result is true for $m=4$. Assume $m \geq 5$. Let $A \in \operatorname{Avoid}\left(m,\left\{H_{1}, H_{2}, H_{3}\right\}\right)$. By our argument in Lemma 5.1, there is a row and a single 
column we can delete, leaving the remainder simple. Let $A^{\prime}$ be the resulting simple matrix. We may assume by induction that there exists disjoint sets $U^{\prime}, L^{\prime}$ such that $\left|U^{\prime}\right|=a \geq 1$, $\left|L^{\prime}\right|=b \geq 1$ where $a+b=m-1$ so that after permuting rows and columns,

$$
A^{\prime}=\begin{gathered}
U^{\prime} \\
L^{\prime}
\end{gathered}\left[\begin{array}{c|c|c|c}
\mathbf{0}_{a} & K_{a}^{1} & \mathbf{0}_{a} & \mathbf{0}_{a} \\
\times & \times & \times & \times \\
\mathbf{1}_{b} & \mathbf{1}_{b} & K_{b}^{b-1} & \mathbf{0}_{b}
\end{array}\right], \text { or } A^{\prime}=U^{\prime}\left[\begin{array}{c|c|c|c}
\mathbf{0}_{a} & K_{a}^{1} & \mathbf{0}_{a} & \mathbf{1}_{a} \\
\times & \times & \times & \times \\
\mathbf{1}_{b} & \mathbf{1}_{b} & K_{b}^{b-1} & \mathbf{1}_{b}
\end{array}\right] .
$$

Without loss of generality, we may assume $b \geq 2$. Assume the last column $(m+2)$ and last row $(m)$ of $A$ is deleted to obtain $A^{\prime}$. After deleting row $m$, the last column of $A$ must be one of the columns of $A^{\prime}$ given that $\left\|A^{\prime}\right\|=\operatorname{forb}\left(m,\left\{H_{1}, H_{2}, H_{3}\right\}\right)$. In order to avoid $H_{1}, H_{2}$ and $H_{3}$ in $A$, we can show that we have the desired structure (5.1) in $A$ with either $a$ or $b$ one larger.

As in the proof of Theorem 1.2, we must do some tedious but straightforward case analysis for which column gets repeated. We move the gory details of the case analysis to Appendix B. Again, this case analysis is much easier to do by oneself than it is to either write or read about.

Proof of Theorem 1.3: We use induction on $m$ for $m \geq 6$. We established by computer that forb $(5, V)=18$ (which is smaller than the bound of Theorem 1.3). Noting that forb $\left(5,\left\{H_{1}, H_{2}, H_{3}\right\}\right)=7$, we deduce using (3.1) that

$$
\operatorname{forb}(6, V) \leq \operatorname{forb}(5, V)+\operatorname{forb}\left(5,\left\{H_{1}, H_{2}, H_{3}\right\}\right)=18+7=25
$$

and so forb $(6, V)=25$, because of construction (5.1). Thus, we may assume $m \geq 7$.

By induction, assume forb $(m-1, V)=\left(\begin{array}{c}m-1 \\ 2\end{array}\right)+\left(\begin{array}{c}m-1 \\ 1\end{array}\right)+\left(\begin{array}{c}m-1 \\ 0\end{array}\right)+3$. Let $A \in \operatorname{Avoid}(m, V)$ with $\|A\|=\operatorname{forb}(m, V)$. Apply the standard decomposition (3.1) to $A$ for some row $r$. If $\left\|C_{r}(A)\right\| \leq m$, we obtain

$$
\begin{aligned}
\|A\| & \leq\left\|C_{r}(A)\right\|+\operatorname{forb}(m-1, V) \\
& \leq m+\left(\begin{array}{c}
m-1 \\
2
\end{array}\right)+\left(\begin{array}{c}
m-1 \\
1
\end{array}\right)+\left(\begin{array}{c}
m-1 \\
0
\end{array}\right)+3 \\
& =\left(\begin{array}{c}
m \\
2
\end{array}\right)+\left(\begin{array}{c}
m \\
1
\end{array}\right)+\left(\begin{array}{c}
m \\
0
\end{array}\right)+3
\end{aligned}
$$

Thus, we may assume that for every $r$ we have $\left\|C_{r}(A)\right\| \geq m+1$. Using Lemma 5.1 (with $m$ replaced by $m-1$ ), we may assume $\left\|C_{r}(A)\right\|=m+1$ for each $r$. Then using Lemma 5.2 we can assume $C_{r}(A)$ has the structure of (5.2) for every $r$ so that every $C_{r}(A)$ partitions $[m] \backslash r$ rows into sets $U_{r}, L_{r}$ with $\left|U_{r}\right|,\left|L_{r}\right| \geq 2$. Note also that the only difference between the two possible structures is a column of 0's or a column of 1's neither of which is used in the case analysis below. Furthermore, we will prove that there is a partition of the rows [m] of $A$ into $U, L$ where $U_{r}=U \backslash r$ and $L_{r}=L \backslash r$.

Take two rows, say $s$ and $t$. Consider $C_{s}(A)$ and $C_{t}(A)$ as determined by (3.1) by setting $r=s$ and $r=t$. Applying Lemma 5.2 when considering $C_{s}(A)$ and $C_{t}(A)$ we 
obtain the partitions $U_{s}, L_{s}, U_{t}, L_{t}$ of rows. Remember that $C_{s}(A)$ and $C_{t}(A)$ both appear twice in $A$ with 0 's and 1's in rows $s$ and $t$ respectively. We now define partitions $U_{s}^{\prime}=U_{s} \backslash t, L_{s}^{\prime} \backslash t, U_{t}^{\prime} \backslash s, L_{t}^{\prime} \backslash s$ so that $U_{s}^{\prime} \cup L_{s}^{\prime}=[m] \backslash\{s, t\}=U_{t}^{\prime} \cup L_{t}^{\prime}$. We assumed $m \geq 7$ and so $|[m] \backslash\{s, t\}| \geq 5$. Hence we may assume that at least one of $U_{s}^{\prime}$ and $L_{s}^{\prime}$ has size at least 3. Without loss of generality, assume $\left|U_{s}^{\prime}\right| \geq 3$.

Let

$$
X=\left[\begin{array}{ll}
1 & 1 \\
1 & 1 \\
0 & 0
\end{array}\right], \quad Y=\left[\begin{array}{ll}
0 & 0 \\
0 & 0 \\
1 & 1
\end{array}\right]
$$

Consider the following three cases:

$\left|U_{s}^{\prime} \cap L_{t}^{\prime}\right| \geq 3$ : We can find $V$ in rows $U_{s}^{\prime} \cap L_{t}^{\prime}$ in $A$ (since $A$ contains two copies of $K_{3}^{1}$ in each triple of rows of $U_{s}^{\prime}$ and two copies of $K_{3}^{2}$ in each triple of rows of $L_{t}^{\prime}$ ).

$\left|U_{s}^{\prime} \cap L_{t}^{\prime}\right|=2$ : Then $\left|U_{s}^{\prime} \cap U_{t}^{\prime}\right| \geq 1$, and so we can find $V$ in $\left.A\right|_{U_{s}^{\prime}}$ by taking two rows of $U_{s}^{\prime} \cap L_{t}^{\prime}$ together with any row in the intersection $U_{s}^{\prime} \cap U_{t}^{\prime}$. We find $Y$ as a submatrix in any row order ( $A$ contains two copies of $K_{3}^{1}$ in each triple of rows of $U_{s}^{\prime}$ ) and we also have $X$ as a submatrix whose first two rows are from $U_{s}^{\prime} \cap L_{t}^{\prime}$ and the last one from $U_{s}^{\prime} \cap U_{t}^{\prime}$. This yields $V$.

$\left|U_{s}^{\prime} \cap L_{t}^{\prime}\right|=1$ : We have $\left|U_{s}^{\prime} \cap U_{t}^{\prime}\right| \geq 2$, and so we can find $V$ in $\left.A\right|_{U_{s}^{\prime}}$ by taking the row of $U_{s}^{\prime} \cap L_{t}^{\prime}$ together with two rows in the intersection $U_{s}^{\prime} \cap U_{t}^{\prime}$. We find $Y$ as a submatrix in any row order ( $A$ contains two copies of $K_{3}^{1}$ in each triple of rows of $U_{s}^{\prime}$ ) and we also have $X$ as a submatrix whose first row is $U_{s}^{\prime} \cap L_{t}^{\prime}$ and last two rows are from $U_{s}^{\prime} \cap U_{t}^{\prime}$. This yields $V$.

This means that $\left|U_{s}^{\prime} \cap L_{t}^{\prime}\right|=0$ and so $U_{s}^{\prime} \subset U_{t}^{\prime}$, but then $\left|U_{t}^{\prime}\right| \geq 3$ and so analogously $U_{t}^{\prime} \subset U_{s}^{\prime}$. So $U_{s}^{\prime}=U_{t}^{\prime}$, and then $L_{s}^{\prime}=L_{t}^{\prime}$. The same conclusion will hold if $\left|L_{s}^{\prime}\right| \geq 3$. Thus for all $s, t \in[m], U_{s}^{\prime}=U_{t}^{\prime}$, and then $L_{s}^{\prime}=L_{t}^{\prime}$.

Using $m \geq 4$, we may now deduce that there is a partition $U, L$ of $[m]$ so that for any $r, U_{r}=U \backslash r$ and $L_{r}=L \backslash r$. This proves that the partition for each $C_{r}$ is really a global partition. Let $|U|=u$ and $|L|=\ell$. We know $u, \ell \geq 2$ since for example if $\left|U_{r}^{\prime}\right| \geq 1$ and $U_{r}^{\prime}=\{s\}$ then $U_{s}^{\prime} \cup\{s\} \subseteq U$ and we have $|U| \geq 2$.

Note that for every row $r$, we have that $\left[\begin{array}{ll}0 & 1\end{array}\right] \times C_{r}(A) \prec A$. We deduce $A$ contains the following columns:

$$
B=\begin{gathered}
U \\
L
\end{gathered}\left[\begin{array}{c|c|c|c|c|c|c|c}
\mathbf{0}_{u} & \mathbf{0}_{u} & \mathbf{0}_{u} & \mathbf{0}_{u} & K_{u}^{1} & K_{u}^{1} & K_{u}^{2} & \mathbf{1}_{u} \\
\times & \times & \times & \times & \times & \times & \times & \times \\
\mathbf{0}_{\ell} & \mathbf{1}_{\ell} & K_{\ell}^{\ell-1} & K_{\ell}^{\ell-2} & K_{\ell}^{\ell-1} & \mathbf{1}_{\ell} & \mathbf{1}_{\ell} & \mathbf{1}_{\ell}
\end{array}\right] .
$$

We have included the column of 0's and the column of 1's since such columns can be added to any matrix without creating $V$. What other columns might we add to this? For $u \geq 3$, matrix $B$ contains

$$
\left[\begin{array}{lll}
1 & 0 & 0 \\
1 & 0 & 0 \\
0 & 1 & 1
\end{array}\right]
$$


in any triple of rows or $U$ in any row order. So $\left.(A-B)\right|_{U}$ must not contain the configuration $(1,1,0)^{T}$, else $A$ has subconfiguration $V$. Similarly for $\ell \geq 3,\left.(A-B)\right|_{L}$ must not contain the configuration $(1,0,0)^{T}$.

Thus, for $u, \ell \geq 3$, all columns of $(A-B)$ are in $\left[\mathbf{0}_{u}\left|K_{u}^{1}\right| \mathbf{1}_{u}\right] \times\left[\mathbf{0}_{\ell}\left|K_{\ell}^{\ell-1}\right| \mathbf{1}_{\ell}\right]$. The only column not already in $B$ is $\mathbf{1}_{u} \times \mathbf{0}_{\ell}$ which is a column of the hypothesized structure (5.1). Thus, without loss of generality, we need only consider the case $u=2, \ell \geq 5$. Let $U=\{a, b\}$ and consider any two rows $c, d \in L$. We know $B$ contains $K_{u}^{1} \times\left[\mathbf{1}_{\ell} \mid K_{\ell}^{\ell-1}\right]$ and $\left[\mathbf{0}_{u} \mid K_{u}^{1}\right] \times K_{\ell}^{\ell-1}$. So $B$ has:

\begin{tabular}{|c|c|c|c|c|}
\hline$a$ & $\begin{array}{ll}0 & 0\end{array}$ & 1 & $\begin{array}{ll}0 & 0\end{array}$ & 1 \\
\hline$b$ & 11 & 0 & & \\
\hline$c$ & $\begin{array}{ll}1 & 1\end{array}$ & 0 & $\begin{array}{ll}0 & 0\end{array}$ & 1 \\
\hline$d$ & & & $\begin{array}{ll}1 & 1\end{array}$ & 0 \\
\hline
\end{tabular}

Note we can interchange $a$ with $b$ and $c$ with $d$. To avoid $V$ we must not have columns in $(A-B)$ with

\begin{tabular}{l|ll}
$a$ & 1 & 1 \\
$b$ & 0 & \\
\hline$c$ & 0 & 1 \\
$d$ & & 0
\end{tabular}.

Thus, the only possible columns of $A-B$ are

\begin{tabular}{c|ccccc}
$a$ & 0 & 1 & 0 & 1 & 1 \\
$b$ & 0 & 0 & 1 & 1 & 1 \\
\hline$L$ & $\alpha$ & $\mathbf{1}_{\ell}$ & $\mathbf{1}_{\ell}$ & $\mathbf{0}_{\ell}$ & $\mathbf{1}_{\ell}$
\end{tabular}.

where $\alpha$ is any column. Recall that since $\ell \geq 3$, any such $\alpha$ must avoid configuration $(1,0,0)^{T}$. All these columns are already in $B$, except for $\mathbf{1}_{2} \times \mathbf{0}_{\ell}$, which together with $B$ completes the hypothesized structure (5.1). The desired bound follows.

Interestingly, the structure of (5.1) falls short of the bound in the case $u=2$.

\section{References}

[1] R.P. Anstee, A Survey of Forbidden Configurations results, http://www . math . ubc . $\mathrm{ca} /$ anstee.

[2] R.P. Anstee, F. Barekat and A. Sali, Small Forbidden Configurations V: Exact Bounds for $4 \times 2$ cases, Studia Sci. Math. Hun., 48(2011), 1-22.

[3] R.P. Anstee, S.N. Karp, Forbidden Configurations: Exact bounds determined by critical substructures, Elec. J. of Combinatorics, 17 (2010), R50, 27pp.

[4] R.P. Anstee and A. Sali, Small Forbidden Configurations IV, Combinatorica , 25(2005), 503-518.

[5] Barricelli, Nils Aall, Symbiogenetic evolution processes realized by artificial methods. Methodos, 9(1957) 143-182. 
[6] Charles Fleurent and Jacques Ferland, Genetic and hybrid algorithms for graph coloring, Annals of Operations Research 63 (1996), 437-461.

[7] A. Kapsalis, V. J. Rayward-Smith, and G. D. Smith, Solving the graphical Steiner tree problem using Genetic Algorithms, The Journal of the Operational Research Society 44 (1993), no. 4, pp. 397-406.

[8] P. Larrañaga, C. M. H. Kuijpers, R.H. Murga, I. Inza, and S. Dizdarevic, Genetic algorithms for the travelling salesman problem: A review of representations and operators, Artificial Intelligence Review 13 (1999), 129-170.

[9] Melanie Mitchell, An introduction to Genetic Algorithms, MIT Press, Cambridge, MA., 1996.

[10] Riccardo Poli, William B. Langdon, and Nicholas Freitag McPhee, A field guide to genetic programming, Lulu Enterprises, UK Ltd, 2008.

\section{A Case analysis for $W$}

Recall that in order to prove Lemma 4.2, only a case analysis for which column was "repeated" is needed. Let $A \in \operatorname{Avoid}\left(m,\left\{G_{1}, G_{2}, G_{3}\right\}\right)$. We deduced that there is a row and a column of $A$ that we can delete and maintain simplicity. Without loss of generality, suppose it is the last row and the last column of $A$. Let $A^{\prime}$ be $A$ without the last row and the last column, which we are assuming by the induction hypothesis to have the desired structure (described in Lemma 4.2). Recall that aside from the column of zeros and the columns of $K_{m-1}^{1}$, there are two columns $\alpha, \beta$ in $A^{\prime}$ with $\alpha<\beta$. We call $\alpha$ the small column and $\beta$ the big column. We consider 3 different types of rows of $A^{\prime}$ :

- Row type 1: Both $\alpha, \beta$ has 0 in the row.

- Row type 2: Column $\beta$ has a 1 and $\alpha$ has a 0 in the row.

- Row type 3: Both $\alpha, \beta$ has 1 in the row.

There may not be any rows of type 1 , but there has to be at least one row of type 2 and at least two rows of type 3. Consider the generic rows below. We've included the appropriate parts of the copy of $K_{m-1}^{1}$ and the column of 0 's. The entries marked $c_{1}, c_{2}, \ldots, r_{4}$ are the entries of the deleted row and column.

\begin{tabular}{ccccccc|c|c} 
& \multicolumn{1}{c}{$\alpha$} & $\beta$ & & \\
\hline 0 & 1 & 0 & 0 & 0 & 0 & 0 & $r_{1}$ & type 1 \\
\hline 0 & 0 & 1 & 0 & 0 & 0 & 1 & $r_{2}$ & type 2 \\
\hline 0 & 0 & 0 & 1 & 0 & 1 & 1 & $r_{3}$ & type 3 \\
0 & 0 & 0 & 0 & 1 & 1 & 1 & $r_{4}$ & type 3 \\
\hline$c_{1}$ & $c_{2}$ & $c_{3}$ & $c_{4}$ & $c_{5}$ & $c_{6}$ & $c_{7}$ & $c_{8}$ &
\end{tabular}.

Of course there might be many rows of each of the types $1,2,3$, but there is no loss of generality if we focus on these rows. There have to be at least two rows of type 3 so it is 
possible to have two rows which correspond to the entries $r_{3}, r_{4}$. We have to be careful because row $r_{1}$ might not exist.

Case 1: $\mathbf{0}_{m-1}$ is the repeated column. Then we have

\begin{tabular}{ccccccc|c}
0 & 1 & 0 & 0 & 0 & 0 & 0 & 0 \\
\hline 0 & 0 & 1 & 0 & 0 & 0 & 1 & 0 \\
\hline 0 & 0 & 0 & 1 & 0 & 1 & 1 & 0 \\
0 & 0 & 0 & 0 & 1 & 1 & 1 & 0 \\
\hline 0 & $c_{2}$ & $c_{3}$ & $c_{4}$ & $c_{5}$ & $c_{6}$ & $c_{7}$ & 1
\end{tabular}

So either $K_{m}^{1} \prec A$ or at least one of $c_{2}, c_{3}, c_{4}, c_{5}$ is 1 . If $c_{2}=1$ then $c_{6}=0$ and $c_{7}=0$ in order to have a laminar family. But then we have $G_{2}$ in the last and next-to-last rows. So we may assume $c_{2}=0$. If $c_{3}=1$ then $c_{5}=0, c_{6}=0$ and $c_{7}=1$ by the laminar property and we have then

\begin{tabular}{lllllll|l}
0 & 1 & 0 & 0 & 0 & 0 & 0 & 0 \\
\hline 0 & 0 & 1 & 0 & 0 & 0 & 1 & 0 \\
\hline 0 & 0 & 0 & 1 & 0 & 1 & 1 & 0 \\
0 & 0 & 0 & 0 & 1 & 1 & 1 & 0 \\
\hline 0 & 0 & 1 & $c_{4}$ & 0 & 0 & 1 & 1
\end{tabular}.

But then we have $G_{2}$ in the last two rows. We may then assume that $c_{3}=0$. If $c_{4}=1$ then both $c_{6}$ and $c_{7}$ have to be 1 , and so we get

\begin{tabular}{lllllll|l}
0 & 1 & 0 & 0 & 0 & 0 & 0 & 0 \\
\hline 0 & 0 & 1 & 0 & 0 & 0 & 1 & 0 \\
\hline 0 & 0 & 0 & 1 & 0 & 1 & 1 & 0 \\
0 & 0 & 0 & 0 & 1 & 1 & 1 & 0 \\
\hline 0 & 0 & 0 & 1 & $c_{5}$ & 1 & 1 & 1
\end{tabular},

which has $G_{3}$ in the last two rows. This completes Case 1.

Case 2: The repeated column has column sum 1. There are three sub-cases, depending on the position of the 1 in the new column. Let $r$ be the row on which, other than the last row, the new column has a 1.

Subcase 2a: $r$ is of type 1 . We have

\begin{tabular}{ccccccc|c}
0 & 1 & 0 & 0 & 0 & 0 & 0 & 1 \\
\hline 0 & 0 & 1 & 0 & 0 & 0 & 1 & 0 \\
\hline 0 & 0 & 0 & 1 & 0 & 1 & 1 & 0 \\
0 & 0 & 0 & 0 & 1 & 1 & 1 & 0 \\
\hline$c_{1}$ & 0 & $c_{3}$ & $c_{4}$ & $c_{5}$ & $c_{6}$ & $c_{7}$ & 1
\end{tabular},

which contains $G_{2}$ in the first two rows. 
Subcase 2b: $r$ is of type 2 . We have

\begin{tabular}{ccccccc|c}
0 & 1 & 0 & 0 & 0 & 0 & 0 & 0 \\
\hline 0 & 0 & 1 & 0 & 0 & 0 & 1 & 1 \\
\hline 0 & 0 & 0 & 1 & 0 & 1 & 1 & 0 \\
0 & 0 & 0 & 0 & 1 & 1 & 1 & 0 \\
\hline$c_{1}$ & $c_{2}$ & 0 & $c_{4}$ & $c_{5}$ & $c_{6}$ & $c_{7}$ & 1
\end{tabular},

which contains $G_{2}$ in the second and third rows.

Subcase 2c: $r$ is of type 3. We have

\begin{tabular}{ccccccc|c}
0 & 1 & 0 & 0 & 0 & 0 & 0 & 0 \\
\hline 0 & 0 & 1 & 0 & 0 & 0 & 1 & 0 \\
\hline 0 & 0 & 0 & 1 & 0 & 1 & 1 & 1 \\
0 & 0 & 0 & 0 & 1 & 1 & 1 & 0 \\
\hline$c_{1}$ & 0 & $c_{3}$ & $c_{4}$ & $c_{5}$ & $c_{6}$ & $c_{7}$ & 1
\end{tabular},

which contains $G_{3}$ in the third and fourth row.

Case 3: The repeated column is the small column $\alpha$. Then we have this:

\begin{tabular}{ccccccc|c}
0 & 1 & 0 & 0 & 0 & 0 & 0 & 0 \\
\hline 0 & 0 & 1 & 0 & 0 & 0 & 1 & 0 \\
\hline 0 & 0 & 0 & 1 & 0 & 1 & 1 & 1 \\
0 & 0 & 0 & 0 & 1 & 1 & 1 & 1 \\
\hline$c_{1}$ & $c_{2}$ & $c_{3}$ & $c_{4}$ & $c_{5}$ & 0 & $c_{7}$ & 1
\end{tabular}.

So $c_{7}$ has to be 1 in order to have a laminar family. If either $c_{4}$ or $c_{5}$ were 0 , then we would have $G_{3}$ in the last row together with one of the next-to-last rows. So both have to be 1 . But this contradicts that we have a laminar family.

Case 4: The repeated column is the big column $\beta$ : Then we have this:

\begin{tabular}{ccccccc|c}
0 & 1 & 0 & 0 & 0 & 0 & 0 & 0 \\
\hline 0 & 0 & 1 & 0 & 0 & 0 & 1 & 1 \\
\hline 0 & 0 & 0 & 1 & 0 & 1 & 1 & 1 \\
0 & 0 & 0 & 0 & 1 & 1 & 1 & 1 \\
\hline$c_{1}$ & $c_{2}$ & $c_{3}$ & $c_{4}$ & $c_{5}$ & $c_{6}$ & 0 & 1
\end{tabular}.

This yields $G_{3}$ in the second and third rows. This completes all cases.

Now if $K_{m}^{1} \prec A$, we readily deduce that $A$ has $\mathbf{0}_{m}$ and for any pair of rows $i, j$, we have $K_{2}^{1}$ in rows $i, j$ of $K_{m}^{1}$. Hence to avoid $G_{2}$, there is no configuration $K_{2}^{1}$ in the remaining columns $\alpha, \beta$ and so we deduce that either $\alpha<\beta$ or $\beta<\alpha$. 


\section{B Case analysis for $V$}

Recall that we had a matrix $A \in \operatorname{Avoid}\left(m,\left\{H_{1}, H_{2}, H_{3}\right\}\right)$ and we deduced there was a row and a column of $A$ we could delete and keep simplicity. Without loss of generality, suppose the deletable row and column are the last row and last column of $A$. We let $A^{\prime}$ be $A$ without the last row and the last column. By the induction hypothesis, we assume $A^{\prime}$ has the desired structure described in Lemma 5.2.

We can make a few general comments about row $m$, the deletable row of $A$. If we have both a 0 and a 1 in row $m$ under the columns containing $K_{a}^{1}$, then using the two columns containing the 0 and 1 and two rows of the $U^{\prime}$ (we may assume $a \geq 2$ in this case because of the two columns) together with row $m$, we obtain a copy of $H_{1}$, a contradiction. Similarly we cannot have both a 0 and a 1 in row $m$ under the columns containing $K_{b}^{b-1}$.

It is also true that in row $m$ we cannot have a 0 under $K_{a}^{1}$ and a 1 under $K_{b}^{b-1}$ else we find a copy of $H_{1}$ in two columns containing 0 and 1 in row $m$ and in a row of $U^{\prime}$, a row of $L^{\prime}$ and row $m$.

We will consider the two cases that $A^{\prime}$ has either the column $\mathbf{0}_{m-1}$ or $\mathbf{1}_{m-1}$ together. Note that for $a=1$, then $A^{\prime}$ has both $\mathbf{0}_{m-1}$ and $\mathbf{1}_{m-1}$.

Let $\gamma$ be the last column of $A$, which we are assuming that, except for row $m, \gamma$ is exactly equal to some other column of $A^{\prime}$. We analyze all possibilities for $\gamma$.

Case 1: $\gamma=\mathbf{0}_{a} \times \mathbf{1}_{b}$. We deduce that column $\mathbf{0}_{m-1}$ of $A^{\prime}$ (if present) appears with a 0 in row $m$ else we have a copy of $H_{1}$ in two rows of $L^{\prime}$ and row $m$. Similarly, if column $\mathbf{1}_{m-1}$ is present then $a \geq 2$ and appears with a 1 in row $m$ else we have a copy of $H_{1}$ in two rows of $U^{\prime}$ and row $m$.

Now if we have 1 's in row $m$ under both $K_{a}^{1}$ and $K_{b}^{b-1}$, then we have the desired structure with $U=U^{\prime}$ and $L=L^{\prime} \cup\{m\}$. Similarly, if we have 0's in row $m$ under both $K_{a}^{1}$ and under $K_{b}^{b-1}$ then we have the desired structure with $U=U^{\prime} \cup\{m\}$ and $L=L^{\prime}$.

Thus we may assume we have 1 's under $K_{a}^{1}$ and 0 's under $K_{b}^{b-1}$. We have two subcases.

Subcase 1a: $A^{\prime}$ has $\mathbf{0}_{m-1}$ (and so $A$ has $\mathbf{0}_{m}$ ). We find a copy of $H_{3}$ in $A$ in a row of $L^{\prime}$ and row $m$ in the column $\mathbf{0}_{m}$ of $A$, a column from $\mathbf{0}_{a} \times K_{b}^{b-1} \times 0_{1}$ with a 0 in the chosen row of $L^{\prime}$, the column with $\mathbf{0}_{a} \times \mathbf{1}_{b} \times \mathbf{0}_{1}$ and a column from $K_{a}^{1} \times \mathbf{1}_{b+1}$.

Subcase 1b: $A^{\prime}$ has $\mathbf{1}_{m-1}$ (and so $A$ has $\mathbf{1}_{m}$ ). If $a=1$ we have that $A^{\prime}$ has $\mathbf{0}_{m-1}$ and so we use Subcase 1a. Assuming $a \geq 2$ we find a copy of $H_{3}$ in $A$ in a row of $U^{\prime}$ and row $m$ in the column $\mathbf{1}_{m}$ of $A$, a column from $K_{a}^{1} \times \mathbf{1}_{b+1}$ with a 1 in the chosen row of $U^{\prime}$, the column with $\mathbf{0}_{a} \times \mathbf{1}_{b} \times \mathbf{0}_{1}$ and a column from $\mathbf{0}_{a} \times K_{b}^{b-1} \times 0_{1}$.

This completes Case 1. 
Case 2: $\gamma=\mathbf{0}_{a} \times \beta_{b}$, where $\beta_{b} \in K_{b}^{b-1}$. Given that $\mathbf{0}_{a} \times \beta_{b}$ is repeated in $A$ on rows $[m-1]$, we deduce that it appears both with a 1 and with a 0 on row $m$. Assume any other column $\mathbf{0}_{a} \times \beta_{b}^{\prime}$ of $A^{\prime}$ (with $\beta_{b}^{\prime} \in K_{b}^{b-1}$ ) has a 0 in row $m$. Then we find a copy of $H_{1}$ in row $m$ and the two rows of $L^{\prime}$ where $\beta_{b}$ and $\beta_{b}^{\prime}$ differ and in the column of $A$ containing $\mathbf{0}_{a} \times \beta_{b}^{\prime}$ and one of the two columns of $A$ containing $\mathbf{0}_{a} \times \beta_{b}$ which differs from the column containing $\mathbf{0}_{a} \times \beta_{b}^{\prime}$ in row $m$. If all entries of row $m$ under $K_{b}^{b-1}$ are 1 , then we find $H_{1}$ by choosing column $\gamma$ and any other column in $\mathbf{0}_{a} \times K_{b}^{b-1}$, together with the corresponding rows in $L^{\prime}$ (the one where $\gamma$ has a 0 and the one where the other column has a 0 ), together with row $m$.

Case 3: $\gamma=\alpha_{a} \times \mathbf{1}_{b}$, where $\alpha_{a} \in K_{a}^{1}$. For $a \geq 2$ we may follow the argument of the previous case and find a copy of $H_{1}$. Given $a=1$, we have that $A^{\prime}$ contains $\mathbf{0}_{m-1}$ as well as $\alpha_{a} \times \mathbf{1}_{b}=\alpha_{1} \times \mathbf{1}_{m-2}=\mathbf{1}_{m-1}$. We deduce that $A$ has $\mathbf{1}_{m}$ and $\mathbf{1}_{m-1} \times \mathbf{0}_{1}$. Thus we can find a copy of $H_{3}$ in a row of $U^{\prime}$ together with a row of $L^{\prime}$, in the two columns with $\mathbf{1}_{m-1}$ in $A^{\prime}$, the column with $\mathbf{0}_{m-1}$ in $A^{\prime}$ and a column of $\mathbf{0}_{1} \times K_{b}^{b-1}$ selected in order to have a 0 on the chosen row of $L^{\prime}$.

Case 4: $\gamma=\mathbf{0}_{a} \times \mathbf{0}_{b}$. We find $H_{3}$ in two rows of $L^{\prime}$ if $b \geq 2$.

Case 5: $\gamma=\mathbf{1}_{a} \times \mathbf{1}_{b}$. If $a \geq 2$, we can find $H_{3}$ in two rows of $U^{\prime}$. In case $a=1$, we know $\mathbf{0}_{m-1}$ as well as $\mathbf{1}_{m-1}$ are in $A^{\prime}$. We find a copy of $H_{3}$ using a row of $U^{\prime}$ and a row of $L^{\prime}$ where we choose a column of $\mathbf{0}_{a} \times K_{b}^{b-1}$ that has a 0 in the chosen row of $L^{\prime}$.

This completes the proof of Lemma 5.2. 